\title{
On the Cool Side: Modeling the Atmospheres of Brown Dwarfs and Giant Planets
}

\author{
M. S. Marley ${ }^{1}$, and T. D. Robinson ${ }^{1}$ \\ 1 NASA Ames Research Center, MS 245-3, Moffett Field, CA 94035, USA
}

\section{Keywords}

atmospheres, brown dwarfs, extrasolar planets, radiative transfer, modeling, convection, chemistry, clouds, opacity

\section{Abstract}

The atmosphere of a brown dwarf or extrasolar giant planet controls the spectrum of radiation emitted by the object and regulates its cooling over time. While the study of these atmospheres has been informed by decades of experience modeling stellar and planetary atmospheres, the distinctive characteristics of these objects present unique challenges to forward modeling. In particular, complex chemistry arising from molecule-rich atmospheres, molecular opacity line lists (sometimes running to 10 billion absorption lines or more) multiple cloud-forming condensates, and disequilibrium chemical processes all combine to create a challenging task for any modeling effort. This review describes the process of incorporating these complexities into one-dimensional radiativeconvective equilibrium models of sub-stellar objects. We discuss the underlying mathematics as well as the techniques used to model the physics, chemistry, radiative transfer, and other processes relevant to understanding these atmospheres. The review focuses on the process of the creation of atmosphere models and briefly presents some comparisons of model predictions to data. Current challenges in the field and some comments on the future conclude the review. 


\section{Introduction}

The atmosphere of a brown dwarf or giant planet, despite constituting a negligible fraction of the total mass, plays a crucial role in controlling the evolution and appearance of the object. By connecting the deep, convective interior with the thermal radiation pouring out from the object, the atmosphere regulates how quickly the interior can cool over time. The atmosphere also imprints the varied signatures of gases, condensates, gravity, and the temperature profile onto emitted thermal radiation, thereby controlling the spectral signature of the object. Thus, understanding the spectrum and evolution over time of a giant planet or brown dwarf requires a working knowledge of the atmosphere. Especially for freely floating brown dwarfs, almost everything we know about an object depends upon our ability to understand its atmosphere.

The atmospheres of brown dwarfs and giant planets, however, are complex. Because these bodies are relatively cool (by stellar standards), chemical equilibrium favors the formation of molecules which often have opacities that vary strongly with wavelength. In addition, at these temperatures condensates can form, adding the seemingly intractable complexity of cloud physics to the problem. Unlike stellar atmospheres, whose 'photosphere' (or the region over which thermal optical depth is near unity) is typically well defined, the strongly wavelength-dependent opacity in brown dwarf and giant planet atmospheres leads to a photosphere that varies with wavelength, and whose physical location can vary by several pressure scale heights.

The aim of this review is to discuss the techniques, and challenges, related to the construction of model atmospheres for brown dwarfs and young giant planets. For specificity, we will consider those processes which influence the atmospheres of the L, T, and Y dwarf spectral types as well as the directly-imaged planets. We will explore influences on the thermal structure, composition, and energy transport in these objects. While our focus is primarily on self-luminous objects, we will also briefly consider the problem of absorption of incident light, relevant to worlds orbiting a primary star.

In this review we will consider the problem of constructing a self-consistent, onedimensional atmospheric structure model that converts a given internal heat flux from the deep, convective interior to radiation that departs the top of the atmosphere. This type of model is usually termed a 'radiative-convective' model, and aims to represent, as an average, the complex three-dimensional structure of the atmosphere. We will explore how gravity, atmospheric composition, gaseous and cloud opacity, and incident flux all influence the thermal profile and spectra of emitted radiation. We will demonstrate the utility such models have for the interpretation of observations, and also consider their limitations for studying complex problems, including time variability and atmospheric dynamics.

A number of previous reviews have covered topics related to radiative-convective modeling of planetary and sub-stellar atmospheres. Of historical interest are reviews by Pecker (1965), who discusses modeling of stellar atmospheres, and Vardya (1970), who examines properties and modeling of low-mass stars. Several decades after these reviews, Allard et al. (1997) revisited models and spectroscopic properties of very low mass stars. These authors also discussed early work in the modeling of brown dwarfs, including grain formation and opacity. Of course, techniques and data for the modeling of condensates, gas and grain opacities, and radiative transfer have progressed substantially in the intervening years.

As the atmosphere controls the cooling rate of the interior, an understanding of brown dwarf or giant exoplanet evolution crucially depends upon the construction of realistic, non-gray atmosphere models for describing the surface boundary condition (Saumon et al. 
1994. Chabrier \& Baraffe 1997). The evolution of very low mass stars and brown dwarfs is reviewed by Burrows et al. (2001), while Saumon \& Marley (2008) present more recent calculations. Burrows et al. (2001) review the data and techniques for computing atmospheric chemistry, abundances, and opacities, compare several cloud models, and review exoplanet and brown dwarf evolution and spectral properties. Kirkpatrick (2005) more completely discusses $\mathrm{L}$ and $\mathrm{T}$ dwarf colors and spectra and the classification of such objects.

The theory of giant planets, emphasizing interior structure, evolution, and what we have learned from studying Jupiter and Saturn, is explored by Hubbard et al. (2002). Very recently, Helling \& Casewell (2014) have reviewed the current state of brown dwarf observations, and discuss related modeling with an emphasis on clouds, including a comparison of a number of cloud models. Finally, Catling (2014) reviews the physics of planetary atmospheres, with an emphasis on Solar System worlds, and the equations and processes that govern atmospheric composition, chemistry, thermal structure, radiative transfer, and circulation.

One reason that the study of cool atmospheres is especially interesting is that it lies at the frontier of two fields: astronomy and planetary science. Indeed among the early theoretical examinations of the first indisputable brown dwarf, Gliese 229B, one set of models (Marley et al. 1996) had a heritage traceable to studies of the atmospheres of Titan and Earth, while two others (Tsuji et al. 1996, Allard et al. 1996) relied on modified stellar atmospheres codes. This convergence of theory from both hot stellar atmospheres and cold planetary atmospheres towards what had once been a non-man's land of atmospheric structure theory (in the realm of effective temperatures of around 500-2500 K) has enhanced the field and provided valuable checks and balances to the theoretical development. Now mostly explored, this review aims to provide a guide for exploration of this new and interesting territory.

We begin this review with an overview of the physics that govern radiative-convective modeling of brown dwarfs and giant planets (Section 22. Following this, we discuss, in turn, the processes that are central to constructing a proper one-dimensional physical model: radiative transfer (Section 3), convection (Section 4), atmospheric chemistry (Section 5), gas opacities (Section 6), and cloud formation and condensate opacities (Section 7). Of course, each of these topics could easily merit their own dedicated review, and the references to classic papers and textbooks cited in this review provide excellent opportunities for more detailed follow-up reading. We, then, outline how these physics are used to actually derive a thermal profile, and present some relevant data-model comparisons. Finally, we conclude with a discussion of some current issues, and prospects for the future.

\section{Physics Overview}

Our focus specifically on atmospheres of brown dwarfs and giant planets simplifies our discussion to the physics of predominantly hydrogen-helium objects, which have been relatively well studied. This review takes the perspective that the atmosphere is a semi-infinite column of gas in hydrostatic equilibrium. The goal is to understand how the gravity, internal heat, energy transport mechanisms, composition, and cloud structures of such an atmosphere influence the thermal profile and, consequently, the properties of its emitted radiation.

Figure 1 shows a schematic of a one-dimensional model atmosphere. The vertical coordinate is pressure, $P$, which is defined on a grid of model levels. In hydrostatic equilibrium, 


\section{COLUMN DENSITIES}

A useful quantity derived using the equation of hydrostatic equilibrium (Equation 1 ) is the column mass, which is the integrated mass per unit area above a given atmospheric level, given by $\mathcal{M}=\int_{z}^{\infty} \rho d z=P(z) / g$. The column molecular number density, $\mathcal{N}$, can be similarly defined, and, using the integral definition, the equation of hydrostatic equilibrium, and assuming an isothermal atmosphere, the column number density is related to the number density profile simply by $\mathcal{N}=n(z) H$. This quantity is especially helpful when estimating optical depths - given an absorption cross section per molecule, $\sigma_{\mathrm{a}}$, the optical depth is roughly $\tau(z)=\sigma_{\mathrm{a}} \mathcal{N}(z)$.

where the gravitational force acting on any given atmospheric slab is balanced by the vertical pressure gradient force, the fluid atmosphere obeys

$$
\frac{d P}{d z}=-g \rho
$$

where $z$ is altitude, $g$ is the acceleration due to gravity, and $\rho$ is the atmospheric mass density. After inserting the ideal gas law and re-arranging, this expression becomes

$$
\frac{d P}{P}=-\frac{g m}{k_{B} T} d z=-\frac{d z}{H}
$$

where $m$ is the mean molecular mass in the atmosphere, $k_{B}$ is Boltzmann's constant $\left(k_{B}=\right.$ $1.381 \times 10^{-16} \mathrm{erg} \mathrm{K}^{-1}$ ), and $T$ is temperature. Here we have also defined the atmospheric pressure scale height, $H=k_{B} T / m g$, which, for an isothermal layer of the atmosphere, is the e-folding distance for pressure, such that the pressure-altitude relation is

$$
P(z)=P\left(z_{0}\right) e^{-\left(z-z_{0}\right) / H},
$$

where $P\left(z_{0}\right)$ and $z_{0}$ are the pressure and altitude at the base of the layer, respectively. Thus, the physical distance between two adjacent model pressure levels shown in Figure 1 can be determined using the layer pressure scale height and Equation 3

Pressure- or altitude-dependent atmospheric properties, such as temperature (shown in Figure 1), chemical composition, or wavelength-dependent thermal flux, are determined either at the model levels or for the model layers (i.e., at the level mid-points). A key model input parameter is the internal heat flux, $F_{\mathrm{i}}$, which, for a non-irradiated world, will set the effective temperature via $\sigma T_{\text {eff }}^{4}=F_{\mathrm{i}}$, where $\sigma$ is the Stefan-Boltzmann constant $\left(5.67 \times 10^{-5} \mathrm{erg} \mathrm{cm}^{-2} \mathrm{~s}^{-1} \mathrm{~K}^{-4}\right)$. In steady state, this energy flux is constant with pressure throughout the atmosphere, and is represented by the dotted region in Figure 1

At great depths in the interior of a brown dwarf or gas giant, the electron density is high and thermal photons cannot propagate far, so energy transport is dominated by convection. Reviews by Stevenson \& Salpeter (1976) and Hubbard \& Smoluchowski (1973) discuss this point. At large ages, very massive brown dwarfs can develop small conductive cores (Lunine et al. 1986, Chabrier et al. 2000), but this is far below the atmosphere. Because convection in these dense atmospheres is very efficient, the gradient in the deep thermal temperature profiles, $\nabla=d \log T / d \log P$, is expected to closely follow convective adiabats (i.e, thermal profiles of constant entropy), with $\nabla=\nabla_{\text {ad }}$ (Baraffe et al. 2002). These

4 Marley E3 Robinson 
assumptions can break down if there are composition gradients that impede convection or deep windows in molecular opacity (e.g., Guillot et al. 1994, Leconte \& Chabrier 2012), which are eventualities that we ignore here.

Convection delivers thermal energy to/through the base of the atmosphere (represented by the blue shaded region at large pressures in Figure 1), and thermal radiative transport (represented by the orange shaded region in Figure 1) begins to become more important as the atmosphere thins with increasing radius (or decreasing pressure). If there are wavelength regions that are both low opacity and which overlap with the local Planck function, then energy can be radiated away through these opacity 'windows'. At some point, as progressively more energy is carried away from the increasingly tenuous atmosphere by radiation, the temperature profile no longer changes as steeply with altitude, indicating that convection has ceased. Above this level, referred to as the 'radiative-convective boundary' (or R-C boundary), energy is carried by radiation, and the atmospheric thermal profile is governed by radiative equilibrium.

In some cases, as the temperature falls with increasing altitude and the peak of the Planck function moves to ever longer wavelengths, this peak may again overlap with a wavelength region of strong opacity. This can again impede thermal radiative energy transport and re-invigorate convection over a small vertical region, called a 'detached convective zone' (see the smaller shaded blue region at lower pressures in Figure 1). Figure 2 better illustrates the physics of such a situation, and shows model profiles of temperature, temperature gradient, and the adiabatic gradient of for a cloud-free late $\mathrm{T}$ dwarf. For this model case a detached convective region is apparent near 1 bar. For four different pressure levels, spectra of the local Planck function, net thermal radiative flux, and absorptivity of the overlying column of gas are also provided. The detached convective region forms when the peak of the local Planck function overlays strong water vapor and methane absorption bands at 2.7 and $3.3 \mu \mathrm{m}$, respectively.

As altitude increases, the atmosphere becomes optically thin to thermal radiation at most wavelengths. However, some strong molecular bands may remain optically thick to fairly high altitudes and will continue to radiate, even while the atmosphere is in general optically thin. For this reason the equilibrium radiative profile is not the same as that expected for a gray atmosphere (i.e., an atmosphere where the opacity is treated as a wavelengthindependent quantity), which reaches a constant-with-altitude 'skin temperature' (for a more detailed discussion, see Pierrehumbert 2010, p. 298).

If the atmosphere is irradiated (i.e., receiving energy from a parent star), then thermal radiative transport and convection must carry the internal heat flux as well as the net absorbed stellar flux. In Figure 1, this is shown conceptually by the vertically-striped flux profile on the right side. For Jupiter, the internal and absorbed incident fluxes are about the same (Hanel et al. 1981). In the case of Uranus the internal flux is vanishingly small (Pearl et al. 1990), while for the so-called 'hot Jupiters' the incident flux dominates completely over the internal contribution.

For a given atmosphere, the internal heat flux and profile of net absorbed stellar flux, along with the opacity structure, control the thermal structure. The equilibrium temperature profile obeys

$$
F_{\mathrm{t}}^{\mathrm{net}}(P)+F_{\mathrm{c}}(P)+F_{\odot}^{\mathrm{net}}(P)-F_{\mathrm{i}}=0,
$$

where $F_{\mathrm{t}}^{\text {net }}$ is the net thermal flux (defined below in Equation 6), $F_{\mathrm{c}}$ is the convective flux, $F_{\odot}^{\text {net }}$ is the net stellar flux, and, as before, $F_{\mathrm{i}}$ is the internal heat flux. Methods for computing these flux profiles, and for determining the thermal structure that satisfies 
Equation 4, are the focus of this review.

\section{Radiation}

A central problem in understanding the equilibrium thermal structure of a sub-stellar atmosphere is to understand energy transport by radiation through the atmosphere. For irradiated bodies, the absorption of stellar flux throughout the atmosphere and/or at the surface drives the climate physics that determine thermal structure. All worlds lose energy by emitting thermal radiation, and thermal radiative transport of energy occurs throughout all atmospheres. In this section we discuss various aspects of this problem, focusing on expressions for the radiative energy fluxes.

\subsection{Radiative Transfer}

Heating and cooling by radiative transfer occurs due to a gradient in the net radiative energy flux,

$$
q_{r}=\frac{g}{c_{P}} \frac{d F_{r}^{\mathrm{net}}}{d P}
$$

where $q_{r}$ is the radiative heating rate (in, for example, $\mathrm{K} \mathrm{s}^{-1}$ ), $c_{P}$ is the local atmospheric heat capacity, and $F_{r}^{\text {net }}$ is the net radiative flux density (typically referred to as just the 'radiative flux'). The net radiative flux is partitioned between an upwelling stream (i.e., towards lower pressures) and a downwelling stream (i.e., towards higher pressures), with

$$
F_{r}^{\text {net }}=F_{r}^{+}-F_{r}^{-}
$$

where a "+" indicates upwelling and a "_" indicates downwelling. Note that the total radiative heating rate is determined from the bolometric net flux, with

$$
F_{r}^{\text {net }}=\int_{0}^{\infty} F_{r, \nu}^{\text {net }} d \nu
$$

where $F_{r, \nu}^{\text {net }}$ is the spectrally resolved net flux. Thus, in the absence of biases in the calculations, uncertainties in the spectrally resolved fluxes at a particular frequency or wavelength can cancel those at other frequencies. A common issue for thermal radiative transport models, though, is that, in very opaque regions of the atmosphere, the upwelling and downwelling fluxes approach the same value, so that the net thermal flux is calculated as the difference between two typically large and similar numbers, requiring high accuracy in the computation of $F_{r}^{+}$and $F_{r}^{-}$.

Most radiative transfer models, in one way or another, solve the 1-D, plane-parallel radiative transfer equation (RTE),

$$
\mu \frac{d I_{\nu}}{d \tau_{\nu}}=I_{\nu}\left(\tau_{\nu}, \mu, \phi\right)-S_{\nu}\left(\tau_{\nu}, \mu, \phi\right)
$$

where $I_{\nu}$ is the spectral radiance, $\tau_{\nu}$ is the frequency-dependent extinction optical depth (which increases towards higher pressures), $\mu$ is the cosine of the zenith angle, $\phi$ is the azimuth angle, and $S_{\nu}$ is the 'source function'. Optical depth can be determined from the absorption coefficient (see Section 6, $k_{\nu}$ (in units of $\mathrm{cm}^{2} \mathrm{~g}^{-1}$, or equivalent), via the differential relation

$$
d \tau_{\nu}=-k_{\nu} \rho_{a} d z
$$

Marley $\&$ Robinson 
where $\rho_{a}$ is the mass density of the absorber (and absorbers simply combine linearly in $\left.k_{\nu} \rho_{a}\right)$. The source function, $S_{\nu}$, is given by

$$
\begin{aligned}
S_{\nu}\left(\tau_{\nu}, \mu, \phi\right)= & \omega_{\nu} F_{\nu}^{\odot} e^{-\tau_{\nu} / \mu \odot} \cdot p_{\nu}\left(\tau_{\nu}, \mu, \phi,-\mu_{\odot}, \phi_{\odot}\right) / 4 \pi \\
& +\left(1-\omega_{\nu}\right) B_{\nu}\left(T\left(\tau_{\nu}\right)\right) \\
& +\omega_{\nu} \int_{0}^{2 \pi} d \phi^{\prime} \int_{-1}^{1} d \mu^{\prime} \cdot I_{\nu}\left(\tau_{\nu}, \mu^{\prime}, \phi^{\prime}\right) p_{\nu}\left(\tau_{\nu}, \mu, \phi, \mu^{\prime}, \phi^{\prime}\right) / 4 \pi
\end{aligned}
$$

where $\omega_{\nu}=\omega_{\nu}\left(\tau_{\nu}\right)$ is the frequency-dependent single scattering albedo, $F_{\nu}^{\odot}$ is the topof-atmosphere solar (or, more generally, stellar) irradiance, $\mu_{\odot}$ is the solar zenith angle, $\phi_{\odot}$ is the solar azimuth angle, $p_{\nu}$ is the scattering phase function, $B_{\nu}$ is the Planck function, and $T\left(\tau_{\nu}\right)$ is the atmospheric temperature profile. The final term on the right-hand side of Equation 10 which represents scattering from directions $\left(\mu^{\prime}, \phi^{\prime}\right)$ into the beam at $(\mu, \phi)$, complicates radiative transfer calculations, as it turns the radiative transfer equation into an integro-differential equation. Note that in the planetary literature it is common to split radiative transfer calculations into solar/stellar and thermal components, wherein the source function would then omit the second and first terms on the right-hand side of Equation 10 respectively. In the case of hot Jupiters there can be substantial wavelength overlap between the thermal emission and incident flux although they have very different angular distributions. The upwelling and downwelling fluxes are related to the angle-dependent radiances by

$$
\begin{gathered}
F_{\nu}^{+}\left(\tau_{\nu}\right)=\int_{0}^{2 \pi} \int_{0}^{1} I_{\nu}\left(\tau_{\nu}, \mu, \phi\right) \mu d \mu d \phi \\
F_{\nu}^{-}\left(\tau_{\nu}\right)=-\int_{0}^{2 \pi} \int_{-1}^{0} I_{\nu}\left(\tau_{\nu}, \mu, \phi\right) \mu d \mu d \phi .
\end{gathered}
$$

A pair of boundary conditions are needed to solve the radiative transfer equation, and these typically specify the downwelling radiation field at the top of the model atmosphere and the upwelling radiation field at the bottom of the atmosphere. For irradiated bodies, the top-of-atmosphere boundary condition for incident radiation is simply a direct or diffuse stellar flux, and the bottom-of-atmosphere boundary condition is that either no flux returns from the deep atmosphere, or that the deep atmosphere has a specified albedo that reflects back some small radiative flux. Thermal calculations assume zero downwelling flux at the top of the atmosphere. The thermal bottom-of-atmosphere boundary condition is less obvious, as the atmospheric column is assumed infinitely deep, but models usually take (Mihalas 1970, p. 165),

$$
I_{\nu}\left(\tau_{\nu}=\tau_{\nu}^{*}, \mu, \phi\right)=B_{\nu}\left(T\left(\tau_{\nu}^{*}\right)\right)+\left.\mu \frac{d B_{\nu}}{d \tau_{\nu}}\right|_{\tau_{\nu}^{*}} \forall \mu>0
$$

where the gradient term allows some flux from deeper layers to contribute at the bottom model level (where $\tau_{\nu}=\tau_{\nu}^{*}$ ), and the factor of $\mu$ ensures that near-vertical streams 'see' deeper into the atmosphere at the boundary.

\subsection{Approaches to Solving the RTE}

Solving the RTE requires a certain level of parameterization or simplification. Most commonly, techniques are divided into either two-stream or multi-stream solutions, where a 'stream' refers to a particular azimuth-zenith coordinate through the atmosphere. Solutions in the two-stream category are more computationally efficient than multi-stream calculations, and offer analytic results that can help to provide insight into problems. Multi-stream calculations provide more detailed information about the angular distribution of intensities and, thus, can provide more accurate solutions for radiant fluxes. 


\section{GRAY RADIATIVE EQUILIBRIUM}

The non-scattering, gray two-stream equations of thermal radiative transfer,

$$
\begin{gathered}
\frac{d F_{t}^{+}}{d \tau}=D\left(F_{t}^{+}-\sigma T^{4}\right) \\
\frac{d F_{t}^{-}}{d \tau}=-D\left(F_{t}^{-}-\sigma T^{4}\right),
\end{gathered}
$$

where $D$ is the diffusivity factor (i.e, a constant that accounts for the integration of intensity over the hemisphere, with preferred values ranging from 1.5-2) and $\tau$ is the gray optical depth, which is related to a gray opacity, $k$, via $d \tau=k d P / g$, can be combined into a single differential equation,

$$
\frac{d^{2} F_{t}^{\mathrm{net}}}{d \tau^{2}}-D^{2} F_{t}^{\mathrm{net}}=-2 D \sigma \frac{d T^{4}}{d \tau}
$$

For a non-irradiated object with an atmosphere in radiative equilibrium, the net thermal flux is constant and equal to $\sigma T_{\text {eff }}^{4}$, so that the previous differential equation can be solved to yield the thermal structure,

$$
T(\tau)^{4}=\frac{1}{2} T_{\mathrm{eff}}^{4}(1+D \tau)
$$

Given either a database of gray opacities, or by making assumptions about the pressure-dependence of $k$, the temperature profile as a function of pressure can be determined. Simple, analytic expressions for the case where convection is included are derived in Robinson \& Catling (2012).

Two-stream solutions have roots in early theories of stellar atmospheres (Schwarzschild 1906), and provide techniques for rapidly computing $F_{\nu}^{+}$and $F_{\nu}^{-}$(which are the two streams). As discussed by Meador \& Weaver (1980), by making assumptions about the scattering phase function and the distribution of intensity in azimuth and zenith angles, the RTE can be simplified to the two-stream equations:

$$
\begin{gathered}
\frac{d F_{\nu}^{+}}{d \tau_{\nu}}=\gamma_{1} F_{\nu}^{+}-\gamma_{2} F_{\nu}^{-}-S_{\nu}^{+} \\
\frac{d F_{\nu}^{-}}{d \tau_{\nu}}=\gamma_{2} F_{\nu}^{+}-\gamma_{1} F_{\nu}^{-}+S_{\nu}^{-}
\end{gathered}
$$

where the frequency-dependent $\gamma$-coefficients permit scattering from the upwelling stream into the downwelling stream and vice versa, and $S_{\nu}^{+/-}$are level-dependent source terms. A critical analysis of the accuracy of different two-stream implementations is also provided by Meador \& Weaver (1980) for cases without internal thermal sources.

A widely-used approach to solving the two-stream RTE with both solar and thermal sources is provided by Toon et al. (1989) (which regrettably contains numerous typographical errors). These authors describe a numerically stable matrix-based solution to the twostream problem. Additionally, to more accurately solve for the thermal radiation field in the presence of scattering, Toon et al. (1989) develop an approach called the 'two-stream source function' technique. Using the solution for the two-stream fluxes, direction-independent scattering source functions, $S_{\nu}^{+/-}$, are determined. These source functions are then used in a multi-stream calculation, where the intensities from the different streams are integrated

$8 \quad$ Marley ES Robinson 
(i.e., via Equation 11 to more accurately determine the upwelling and downwelling fluxes. The approach is accurate in most cases excepting situations with very high albedo, very highly foward scattering particles. Thus, the two-stream source function approach represents an efficient middle ground for computing the atmospheric energy balance and thermal profile between strict two-stream solutions and general multi-stream solutions, and is the approach currently used in the Marley/Saumon brown dwarf and giant planet models (e.g.,

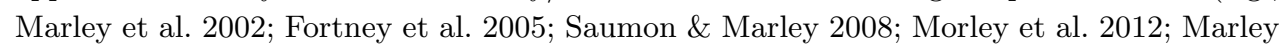
et al. 2012).

True multi-stream solutions seek to solve the full RTE with self-consistent multiple scattering. Such solutions commonly solve the azimuth-independent form of the RTE (Chandrasekhar 1960, p. 15), as aerosol scattering phase functions are typically expressed in terms of a single angle - the scattering angle. More general techniques have been developed for solving the azimuth-dependent RTE, which are necessary when, for example, the solar/stellar source comes from a particular azimuth and zenith angle (Milkey et al. 1975 Stamnes et al. 1988).

Numerical techniques for determining angle-dependent intensities along a set of discrete zenith angles (representing the streams) in scattering and/or emitting atmospheres/media have been developed in both the planetary and astrophysical literatures. For the latter, the Accelerated Lambda Iteration (ALI) approach (Cannon 1973. Olson et al. 1986 Hubeny \& Lanz 1992, Hauschildt 1992) is most common (see also review by Hubeny 2003). In ALI, the level- and frequency-dependent source function $\left(S_{\nu}\right)$ is iteratively adjusted, with each new iteration providing intensities that are increasingly accurate solutions to the RTE. This technique is currently used in the PHOENIX atmospheric models Allard \& Hauschildt 1995. Barman et al. 2001), and in the TLUSTY radiative transfer model (Hubeny \& Lanz 1995 ) implemented in models by Burrows et al. (Burrows et al. 2002).

Multi-stream techniques for solving the RTE developed in the Earth and planetary literature include adding-doubling and discrete ordinates. In the former, optically thin, homogeneous atmospheric layers - with given absorbing, emitting, and scattering properties - are combined to form an inhomogeneous atmospheric model (van de Hulst 1963, Twomey et al. 1966). A set of recursive relations, based on the linear interaction of the radiation with a thin layer, are used to compute the intensities within the inhomogeneous model (Hansen 1969 Wiscombe 1976 Evans \& Stephens 1991). The discrete ordinates method, originally developed by Chandrasekhar (1960, p. 56), recasts the RTE as a system of ordinary differential equations and uses matrix techniques to find a solution to the system (Stamnes \& Swanson 1981). This method is used in the popular, and publicly available, radiative transfer model DISORT (Stamnes et al. 1988, 2000).

\section{Convection}

Absorption of stellar flux at a planetary surface or deep in the atmosphere of a gaseous world, or the presence of a large internal heat flux, can lead to thermal structures that are unstable to vertical convection. Here, a parcel of gas that is displaced upwards would find itself in an environment whose density is greater than the parcel's internal density, so the parcel would continue to rise (or parcels displaced downwards continue to sink). This instability leads to a critical vertical density gradient in an atmosphere, with convection occurring when the gradient is too steep. Using an equation of state, this density gradient can be related to a temperature gradient (or "lapse rate"), $-d T / d z$, that defines the limit 
between thermal structures that are convectively stable versus unstable.

Fortunately, modeling convection in brown dwarf and giant planet atmospheres is more straightforward than in the stellar structure literature (e.g., Hansen et al. 2004. Chapter 5), since the ideal gas law applies and the convection is, to a good approximation, adiabatic (Baraffe et al. 2002, Ludwig et al. 2006, Freytag et al. 2010). Given these, the criterion for an unstable lapse rate is

$$
-\frac{d T}{d z}>\frac{g}{c_{P}}
$$

or, by including the equation of hydrostatic equilibrium,

$$
\nabla=\frac{d \log T}{d \log P}>\frac{R_{s}}{c_{P}}=\nabla_{\mathrm{ad}}
$$

where $R_{s}$ is the specific gas constant, which is equal to $k_{B} / m$. Thus, for lapse rates larger than the "dry adiabatic lapse rate", $g / c_{P}$ (or $R_{s} / c_{P}$ in $d \log T / d \log P$ ), a parcel of gas that is perturbed upwards will continue to rise, meaning that convection would ensue.

It is important to remember that convection does not create or destroy energy - it simply redistributes heat. Thus, convection schemes should be energy conserving. Since lapse rates in a convectively unstable atmosphere are larger than the adiabatic lapse rate, convection will work to move heat from deeper atmospheric levels to levels at lower pressures (higher altitudes). In this regard, convection serves to limit the thermal contrast between the deep atmosphere and the radiative-convective boundary.

Approaches to modeling convection (an inherently three-dimensional process) in onedimensional thermal structure models are varied. Models rooted in the planetary science literature commonly employ an approach called 'convective adjustment', while those derived from astrophysical sources will tend to use mixing-length theory. These are discussed in turn below.

\subsection{Convective Adjustment}

Convection in brown dwarf and giant planet atmospheres relaxes the thermal structure onto an adiabat (Ludwig et al. 2006), so that the structure throughout the convective portion of the atmosphere can be modeled using the dry adiabatic lapse rate. In their simulations of Earth's atmospheric thermal structure, Manabe \& Strickler (1964) outlined a straightforward scheme for forcing convectively unstable atmospheric layers onto an adiabat, which these authors called 'convective adjustment.' In this work, radiative heating and cooling rates were used to timestep a model atmosphere towards radiative equilibrium. At each timestep, and proceeding upward from the surface, the lapse rate for each model layer, $|\Delta T / \Delta z|$, was compared to an adiabatic lapse rate. If the layer was unstable, then the temperatures at the top and bottom of the layer were immediately changed, in an energyconserving fashion, to give the adiabatic lapse rate. An explicit description of this scheme is given in Manabe \& Wetherald (1967).

For atmospheres experiencing a substantial amount of condensation, latent heat release causes the lapse rate in the convective portion of the atmosphere to be smaller than the dry adiabatic lapse rate. For example, on Earth the dry adiabatic lapse rate is $g / c_{P}=$ $9.8 \mathrm{~K} \mathrm{~km}^{-1}$, whereas the actual average lapse rat $1 \mathrm{n}$ the troposphere is about $6.5 \mathrm{~K} \mathrm{~km}^{-1}$, which is smaller due to water vapor condensation. So, in the original work by Manabe \& Strickler (1964), the convective portion of the atmosphere was simply relaxed to the 


\section{DRY ADIABATIC LAPSE RATES AND TEMPERATURE GRADIENTS}

Combining the dry adiabatic lapse rate, $-d T / d z=g / c_{P}$, and the equation of hydrostatic equilibrium (Equation 1), we find the equivalent of the dry adiabat in pressure-space,

$$
\frac{d T}{d P}=\frac{1}{c_{P} \rho} .
$$

Then, using the ideal gas law to express density in terms of pressure and temperature, we have

$$
\frac{d T}{d P}=\frac{k_{B} T}{m c_{P} P},
$$

or

$$
\nabla_{\mathrm{ad}}=\frac{d \log T}{d \log P}=\frac{R_{s}}{c_{P}}
$$

Recalling from kinetic theory that the specific gas constant is equal to $c_{P}-c_{v}$, where $c_{v}$ is the specific heat at constant volume, and the ratio of specific heats is,

$$
\gamma=\frac{c_{P}}{c_{v}}
$$

we can then write the dry adiabat as simply

$$
\nabla_{\mathrm{ad}}=\frac{\gamma-1}{\gamma}
$$

Thus, over regions of the atmosphere where the internal degrees of freedom of the gas are roughly constant, the temperature-pressure relationship along a dry adiabat is given by Poisson's adiabatic state equation,

$$
T(P)=T_{0}\left(\frac{P}{P_{0}}\right)^{(\gamma-1) / \gamma}
$$

where $T_{0}$ and $P_{0}$ are a reference temperature and pressure, respectively, along the adiabat.

measured lapse rate instead of the dry adiabatic lapse rate.

Of course, for a given background condensible gas, one expects that the influence of latent heating in the convective portion of an atmosphere to be less significant at lower temperatures, as cooler temperatures imply smaller amounts of the condensible. The moist adiabatic lapse rate (Satoh 2004, p. 249) captures these physics by accounting for the effects of latent heat release on a parcel or air that experiences condensation while being lifted adiabatically through the background atmosphere. While multiple species indeed condense in ultracool dwarf atmospheres, in practice the contribution of latent heating is only of first order importance for water clouds, so we forego a deeper discussion of this issue here.

The strength of the convective adjustment approach is that it is computationally

\footnotetext{
${ }^{1}$ According to the U. S. Standard Atmosphere (1976), NOAA-S/T76-1562.
} 
efficient - the thermal structure is simply taken to lie on a dry or moist adiabat. Given this assumed structure, numerical models need only determine the location of the radiativeconvective boundary, and then ensure that temperature and thermal flux are continuous across this boundary.

Convective adjustment has several notable shortcomings beyond the difficulties associated with condensible gases discussed above. First, convective adjustment may not realistically capture the dynamical response of an atmosphere to vertical motions. Super-adiabatic layers are immediately adjusted onto an adiabat, without regard for any vertical mixing timescales. This assumption is justified when only a steady-state solution is required, but could become problematic when studying time-dependent atmospheric variability (see Section 10.1. Additionally, convective adjustment does not straightforwardly predict an eddy diffusion coefficient, which is a critical input to chemistry and cloud models. One potential bypass for this shortcoming is to follow the scaling arguments of Gierasch \& Conrath (1985. Equation 16), who use mixing length theory to derive an expression for the eddy diffusivity.

\subsection{Mixing Length Theory}

Mixing length theory (Prandtl 1925, Vitense 1953, Böhm-Vitense 1958) uses dimensional and scaling arguments to model convective fluxes, and has seen successful applications and development in both the astrophysical and planetary science literatures (Henyey et al. 1965. Gierasch \& Goody 1968 Spiegel 1971, Castelli et al. 1997). In this approach, convection is modeled as the diffusion of heat through a turbulent medium. The turbulent diffusivity is taken as

$$
K_{h}=w l
$$

where $l$ is a characteristic length over which turbulent mixing occurs (i.e., the mixing length), and $w$ is a characteristic upward transport velocity. For a parcel lifted adiabatically through $l$, the temperature difference between the parcel and its surroundings is $\Delta T=$ $-l\left(d T / d z+g / c_{P}\right)$, where $d T / d z$ is the lapse rate for the parcel, so that the convective flux is

$$
F_{c}=w \rho c_{P} \Delta T=-\rho c_{P} K_{h}\left(\frac{d T}{d z}+\frac{g}{c_{P}}\right),
$$

where, of course, a convective heat flux is only present when the atmosphere is unstable to convection (i.e., $-d T / d z>g / c_{P}$ ).

Models usually assume that the mixing length is proportional to the pressure scale height, with $l=\alpha H$, where $\alpha$ is a free parameter, typically of order unity (see Burrows et al. 2001. Baraffe et al. 2002, Robinson \& Marley 2014, for exploration of this parameter). The characteristic transport velocity can be derived from buoyancy force arguments (Kippenhahn et al. 2012, p. 62), yielding $w=l\left[-g / T\left(d T / d z+g / c_{P}\right)\right]^{1 / 2}$. Thus, the turbulent diffusivity in a convectively unstable portion of the atmosphere is given by

$$
K_{h}=l^{2}\left[-\frac{g}{T}\left(\frac{d T}{d z}+\frac{g}{c_{P}}\right)\right]^{1 / 2} .
$$

Mixing length approaches benefit from being simple and computationally efficient. Additionally, these techniques directly compute the convective heat flux and turbulent diffusivity (Equations 17 and 18 respectively). The latter gives information about mixing processes in the convective portion of the atmosphere, and can be used in chemical and aerosol transport models. Also, since convective heating and cooling rates can be computed 


\section{CONVECTIVE EFFICIENCY}

Using the turbulent diffusivity in Equation 18 , we can write the convective flux (Equation 17) as

$$
F_{c}=\rho c_{P} l^{2}\left(\frac{g}{T}\right)^{1 / 2}\left[-\left(\frac{d T}{d z}+\frac{g}{c_{P}}\right)\right]^{3 / 2} .
$$

If we then assume that the atmospheric lapse rate is some fraction larger than the dry adiabatic lapse rate, with

$$
\frac{d T}{d z}=-(1+f) \frac{g}{c_{P}}
$$

and that the mixing length is simply the pressure scale height, then the convective flux is

$$
\begin{aligned}
F_{c} & =\rho c_{P} H^{2}\left(\frac{g}{T}\right)^{1 / 2}\left(f \frac{g}{c_{P}}\right)^{3 / 2} \\
& =f^{3 / 2} R_{s} P\left(\frac{T}{c_{P}}\right)^{1 / 2}
\end{aligned}
$$

where the second step used the ideal gas law and the definition of the pressure scale height. Notably, the gravitational acceleration has cancelled out of this expression.

We can now get a sense for how efficient convection is at returning a super-adiabatic lapse rate to near the dry adiabatic value. In the convective region of a mid-L dwarf $\left(T_{\text {eff }}=1900 \mathrm{~K}, g=100 \mathrm{~m} \mathrm{~s}^{-1}\right)$, with $P=10$ bar, $T=3500 \mathrm{~K}$, and $c_{P}=10^{9} \mathrm{erg} \mathrm{g}^{-1} \mathrm{~K}^{-1}$, and with $R_{s}=4 \times 10^{7} \mathrm{erg} \mathrm{g}^{-1} \mathrm{~K}^{-1}$, we find $F_{c}=7 \times 10^{11} f^{3 / 2}$ erg $\mathrm{cm}^{-2} \mathrm{~s}^{-1}$. This flux is impressively large even for a $1 \%$ deviation from the dry adiabat (i.e., $f=0.01$ ), the convective flux is large enough to carry the entirety of the internal heat flux, or $\sigma T_{\text {eff }}^{4}=7.4 \times 10^{8} \mathrm{erg}^{\mathrm{cm}^{-2}} \mathrm{~s}^{-1}$. Thus, even very minor deviations of the lapse rate from the dry adiabatic value will be promptly eliminated.

from the convective flux profile, mixing length models can be used to study time-dependent atmospheric processes and phenomenon.

A common criticism of mixing length theory is that the mixing length, $l$, is ambiguous. Additionally, the theory does not directly predict the thermal structure in the convective regime (unlike convective adjustment which imposes the adiabat), but, instead, must solve for the thermal structure using the relevant fluxes and heating rates.

\subsection{Beyond One-Dimensional Models of Convection}

Studies of stellar structure and work in the Earth and planetary sciences have benefited from two- and three-dimensional modeling of convection, and from comparing these models to one-dimensional methods for simulating convection. For example, Chan et al. (1982) performed two-dimensional simulations of turbulent convection in the deep atmospheres of stars and noted that the vertical velocities in their models were correlated over a characteristic length comparable to the pressure scale height, which lends some support to the mixing length picture outlined above. Cattaneo et al. (1991) used three-dimensional models to study convection in an astrophysical setting, and noted that local mixing length theories can adequately represent the turbulent transport of energy.

The brown dwarf and irradiated giant planet literature has seen very little develop- 
ment of convection-resolving models. Numerous global circulation models (GCMs) for hot Jupiters (see, e.g., review by Showman et al. 2008) and brown dwarfs (Showman \& Kaspi 2013. Zhang \& Showman 2014) exist. However, only Freytag et al. (2010) have applied convection-resolving models to brown dwarfs in their study of dust transport in cool and sub-stellar atmospheres. Application of models and techniques from the Earth, planetary, and stellar literatures may, then, prove fruitful in advancing our understanding of convection in brown dwarf and giant exoplanet atmospheres.

\section{Chemistry}

In addition to convection and radiative transfer, of course, a key characteristic of an atmosphere is its chemical makeup, which can in turn affect the computation of the thermal structure as gas abundances influence many processes (such as, e.g., opacities and, thus, radiative energy transport). In this section we briefly review influences on gas concentrations and discuss how the atmospheric composition is computed in the context of one-dimensional modeling. More extensive reviews of this topic can be found in Lodders \& Fegley (2006) and Burrows et al. (2001).

\subsection{Abundances}

Of central importance to modeling the chemistry throughout a brown dwarf or giant planet atmosphere are the abundances of the underlying elements that make up the more complex molecules that form in these atmospheres. These abundances are affected by two key processes. First, the overall elemental abundances for the object determine the baseline distribution of elements. Second, the formation and rainout of condensates will influence the availability of certain elements, possibly starving upper atmospheric layers (i.e., those at lower pressures and cooler temperatures) of certain elements.

5.1.1. Elemental. A key assumption in any atmosphere model is the underlying elemental abundances. The most important individual abundances being those of carbon and oxygen $\left(\mathrm{C}\right.$ and $\mathrm{O}$ ), as $\mathrm{H}_{2} \mathrm{O}, \mathrm{CO}$, and $\mathrm{CH}_{4}$ are key absorbers whose concentrations are controlled by the availability of $\mathrm{C}$ and $\mathrm{O}$. Abundances are typically referenced to those of the Sun, and an assumption of 'solar abundances of the elements' is usually the starting point for atmospheric modeling. Asplund et al. (2009) reviews the challenges in defining such an abundance set. Unfortunately the solar $\mathrm{C}$ and $\mathrm{O}$ abundances are uncertain, and the generally accepted values have varied with time, even over the short history of ultracool dwarf modeling (e.g., Anders \& Grevesse 1989, Allende Prieto et al. 2001, 2002, Asplund et al. 2009 Caffau et al. 2011). For this reason any model comparison between different modeling groups must begin with a comparison of the assumed elemental abundances. Barman et al. (2011), for example, utilize the abundances of Asplund et al. (2009) which are very similar to the Lodders (2003) abundances utilized by the Marley/Saumon group.

Not all atmospheres will have solar abundances of course. Individual brown dwarfs or giant planets will sport a variety of compositions, and the defining characteristic of extrasolar giant planets may well be atmospheric compositions that depart from that of their primary stars. Thus any given atmosphere model must make some choice for the initial elemental abundances.

An additional important aspect of abundance determination is the $\mathrm{C} / \mathrm{O}$ ratio, which 
not only affects the relative abundance of the major carbon- and oxygen-bearing species, but other compounds as well. As the $\mathrm{C} / \mathrm{O}$ ratio increases towards unity, the condensation temperatures of oxides and silicates fall and C-bearing compounds become more prevalent (see discussion in Lodders 2003, and references therein). Indeed the accepted 'solar' value of $\mathrm{C} / \mathrm{O}$ has ranged from 0.4 to 0.6 , and is known to vary amongst other stars (for a recent discussion, see Fortney 2012). Thus the assumed model C/O ratio should also always be noted when presenting modeling results. Fortney $(2012)$ has suggested that brown dwarf spectra are especially well-suited to measuring the $\mathrm{C} / \mathrm{O}$ ratio in the solar neighborhood. In the extreme case, if a brown dwarf were to be formed with atmospheric $\mathrm{C} / \mathrm{O}>1$, the entire atmospheric chemistry would be grossly altered, with essentially all available $\mathrm{O}$ going to form $\mathrm{CO}$ instead of $\mathrm{H}_{2} \mathrm{O}$, with the excess $\mathrm{C}$ forming $\mathrm{C}_{2}$ and $\mathrm{HCN}$. However, no such brown dwarfs have yet been found and we neglect this possibility here.

5.1.2. Rainout. A crucial distinction between modeling approaches arises in the treatment of the chemistry of condensates. Once a condensate is formed, two limiting cases can be imagined. In one case the solid grain or liquid drop will continue to chemically interact with the surrounding gas to arbitrarily low temperatures. This is a description of 'true' chemical equilibrium where a given initial set of elements is always assumed to be in chemical equilibrium at a specified temperature and pressure. This assumption was explored by Burrows \& Sharp (1999), and is implicit in the COND and DUSTY models (Chabrier et al. 2000 Allard et al. 2001). In the other extreme, the condensate can be imagined to rain out of the atmosphere, precluding further reactions with the neighboring gas, a limit sometimes called 'rainout chemistry' (although 'sedimentation chemistry' is probably a better phrase, 'rainout' has become ingrained in the literature).

Discussions of rainout and the gas and cloud chemistry in sub-stellar atmosphere can be found in Lodders (1999), Burrows \& Sharp (1999), Lodders (2004), and Lodders \& Fegley (2006). An important consequence of the rainout chemistry is that reactions that would be expected to take place between condensed species and the gas at temperatures cooler than the condensation temperature are excluded. For example, without the rainout assumption, iron grains would react with $\mathrm{H}_{2} \mathrm{~S}$ gas to form $\mathrm{FeS}$, thus removing $\mathrm{H}_{2} \mathrm{~S}$ from the atmosphere at around $500 \mathrm{~K}$. In contrast, under the rainout assumption the iron grains are presumed to fall out of the atmosphere after they form at around $2000 \mathrm{~K}$, thereby precluding further reaction with $\mathrm{H}_{2} \mathrm{~S}$, which then stays in the gas phase. Since $\mathrm{H}_{2} \mathrm{~S}$ is indeed observed in Jupiter's atmosphere, this is presumably the correct limit Barshay \& Lewis 1978, Fegley \& Lodders 1994 Niemann et al. 1998).

Another example of the importance of rainout arises in the alkali chemistry and the interaction of the alkali metals with silicate grains that initially form around $1800 \mathrm{~K}$. Without the rainout assumption, gaseous sodium and potassium undergo a series of reactions with these grains that ultimately produce alkali feldspar $\left([\mathrm{Na}, \mathrm{K}] \mathrm{AlSi}_{3} \mathrm{O}_{8}\right)$, thereby removing $\mathrm{Na}$ and $\mathrm{K}$ from the gas at around $1400 \mathrm{~K}$. With rainout the silicate grains that form near $1800 \mathrm{~K}$ fall, do not react with the atmosphere at cooler temperatures, and alkali feldspar does not form (Lodders 1999). In this limit sodium remains in the atmosphere until about $700 \mathrm{~K}$ where it forms $\mathrm{Na}_{2} \mathrm{~S}$ and $\mathrm{KCl}$. Marley et al. (2002) argue that the far red optical spectra of $\mathrm{T}$ dwarfs support the rainout hypothesis. More recently, Morley et al. (2012) convincingly demonstrate the presence of such alkali condensates in cool $\mathrm{T}$ dwarf spectra. Taken together these lines of evidence support the rainout limit. 


\subsection{Equilibrium Chemistry}

Given an abundance of all relevant elements and appropriate thermodynamic data, the abundances of gaseous species at any pressure and temperature can be computed. Different groups utilize one of primarily two different methods for solving for the gas abundances in chemical equilibrium. The quality of the results ultimately depends upon the number of species considered and the veracity of the underlying thermodynamic data, which are not always available for all reactions and species of interest.

The two methods for finding equilibrium are commonly termed 'mass action' and 'Gibbs minimization.' The mass action approach utilizes the equilibrium constants for all relevant chemical reactions, as well as mass conservation, to find the abundance of each molecule and condensate of interest at a given pressure and temperature. Gibbs minimization solves for the mixture of species that has the lowest Gibbs free energy, given the pressure, temperature, and assumed elemental abundances. With identical thermodynamic data-and the same assumptions regarding rainout - the two approaches should give identical results. However, in practice, typically because of computational limitations, mass conservation is not always achieved by Gibbs minimization methods. Computational considerations also often limit the number of elements and compounds that can be included in this approach. The best source for learning about the details of chemical equilibrium calculations is van Zeggeren \& Storey (1970).

For specific application to brown dwarf atmospheres, Section 4 of Sharp \& Burrows (2007) provides ample discussion and examples of the free-energy minimization procedure. Likewise, the mass action approach is described by Fegley \& Lodders (1994) in the context of Jupiter's atmosphere, and the method has been used to study the chemistry of the major elements in brown dwarf and exoplanet atmospheres by Lodders \& Fegley (2002) and Visscher et al. (2006).

Regardless of the method employed, the curation and vetting of relevant thermodynamic data is a time-consuming process. For example Lodders, Fegley, and collaborators follow approximately 2,000 gaseous and 1,700 solid or liquid species in their code. Construction of the relevant thermodynamic database poses a substantial "barrier to entry" for new scientists interested in computing their own atmospheric chemistry.

Examples of equilibrium gas abundances, or ratios of gas abundances, for several key species of interest (from the rainout models of Lodders \& Fegley 2002 Visscher et al. 2006) are shown in Figure 3 along with model temperature profiles for a few representative objects. The effects of condensation removing Ti and Fe can clearly be seen. Also, the ratios of the concentrations of $\mathrm{CH}_{4}$ to $\mathrm{CO}$ and $\mathrm{NH}_{3}$ to $\mathrm{N}_{2}$ demonstrate the preferred chemical state of carbon and nitrogen at any given temperature and pressure.

\subsection{Disequilibrium Chemistry}

Although Jupiter's atmosphere is primarily in the expected state of chemical equilibrium, its atmospheric composition departs from equilibrium in some important respects, and these provide a guide for understanding similar excursions in brown dwarf and exoplanet atmospheres. First, convection is known to mix CO from the deep atmosphere, where it is the favored carbon-bearing species, up to the observable atmosphere, where $\mathrm{CH}_{4}$ should be overwhelmingly dominant (Prinn \& Barshay 1977, Bézard et al. 2002). This can happen in situations where the mixing time for species to be transported by convection is shorter than the timescale for a species to come into chemical equilibrium with its surroundings. 
Because carbon is tied to oxygen by a strong triple bond in $\mathrm{CO}$, it can be difficult for atmospheric chemical processes to convert the species to methane, even when this would be thermodynamically favored. Figure 4 illustrates the somewhat tortuous chemical pathway linking $\mathrm{CO}$ to $\mathrm{CH}_{4}$. For this reason Fegley \& Lodders (1996) predicted that CO would be discovered in the atmospheres of what were then termed the 'methane dwarfs' (now the $\mathrm{T}$ dwarfs) and, indeed, several observational studies (Noll et al. 1997, Oppenheimer et al. 1998, Saumon et al. 2000, Geballe et al. 2009) found excess CO in these objects. Likewise, excess $\mathrm{CO}$ was found in the atmospheres of the cooler transiting planets and the repeated "discovery" of chemical disequilibrium became, for a while, something of an industry within the exoplanet community.

The efficiency with which $\mathrm{CO}$ can be transported upwards depends both upon the vigor of eddy mixing and by the chemical equilibrium timescale. The former is usually parametrized in models with $K_{h}$, the eddy diffusivity for heat discussed earlier, while the latter depends upon the details of the chemical pathway. The usual approximation used in modeling this process is that the atmospheric abundance of $\mathrm{CO}$ is fixed at the point where the chemical equilibrium timescale is equal to the mixing timescale, commonly known as the 'quench' approximation (e.g., Fegley \& Lodders 1996, Saumon et al. 2000, Lodders \& Fegley 2002, Hubeny \& Burrows 2007). An example is shown in Figure 5 For this model a pure equilibrium calculation would find that carbon in the atmosphere should be almost entirely in the form of methane above about 1 bar when the thermal profile has entered in the methane stability field. However, in the presence of vertical mixing, CO is transported upwards and can be the dominant species when mixing is vigorous. The most comprehensive discussions of this process can be found in Hubeny \& Burrows (2007) and Zahnle \& Marley (2014)

Zahnle \& Marley (2014) review the disequilibrium mixing literature, and argue that this process is more gravity-dependent than previously recognized. They suggest that, in the lowest mass extrasolar planets, such as those that are expected to be discovered by the Gemini Planet Imager (GPI) and the Spectro-Polarimetric High-contrast Exoplanet REsearch (SPHERE) surveys for young planets (Macintosh et al. 2008 Beuzit et al. 2008), CO may be the dominant carbon bearing species until temperatures as low as about $600 \mathrm{~K}$. Furthermore, CO is not the only species that can be in disequilibrium because of mixing. Similar arguments apply to the equilibrium between $\mathrm{N}_{2}$ and $\mathrm{NH}_{3}$, as well as the chemistry of $\mathrm{PH}_{3}$ and $\mathrm{GeH}_{4}$. Indeed signs of $\mathrm{NH}_{3}$ under-abundance compared to the expectations of equilibrium chemistry have already been reported in brown dwarfs (Saumon et al. 2006), which has also been reviewed by Zahnle \& Marley (2014).

One final important disequilibrium process should be noted-under the presence of incident ultraviolet radiation, methane, hydrogen sulfide, and other species may be photochemically destroyed, leading to the creation of more complex molecules. In cool Solar System atmospheres the resulting species often form haze particles which themselves become important opacity sources. A thorough post-Voyager review of methane photochemistry in the context of giant planets is Bishop et al. (1995), while a concise, informative overview of giant planet C, N, O, and S photochemistry can be found in Moses (2000). Zahnle et al. (2009) consider photochemical processes that are unique to giants much warmer than Jupiter, specifically studying sulfur photochemistry in hot Jupiter atmospheres. In general, photochemistry in any giant more heavily irradiated than Jupiter will be much more complex than in Solar System giants since species that are usually trapped in tropospheric clouds, such as $\mathrm{NH}_{3}, \mathrm{H}_{2} \mathrm{~S}$ and $\mathrm{H}_{2} \mathrm{O}$, will instead be present in the upper atmosphere where 


\section{QUENCHING}

Turbulent motions will tend to cause the vertical mixing of chemical species throughout an atmosphere. Chemical disequilibrium can be established if mixing supplies a species to an atmospheric level faster than the local chemical reactions can remove it. The concentration of the species is then locked into its value from deeper in the atmosphere, in a process called 'quenching.' The quenching level is roughly where the mixing timescale equals the chemical loss timescale, or $t_{\text {mix }}=t_{\text {chem }}$.

Given the characteristic turbulent velocity, $w$, mixing over a pressure scale height, $H$, occurs at a timescale

$$
t_{\text {mix }}=\frac{H}{w}=\frac{H^{2}}{K_{h}},
$$

where the second step used Equation 16 with $l=H$ (but see Smith $(1998)$ for an alternative approach to the use of $H$ as well as the discussion in Zahnle \& Marley (2014)). The value of $K_{h}$ can either be evaluated from a thermal structure model using mixing length theory (Section 4.2 , or simply specified as a constant. Above the convective region, the eddy diffusivity from convective mixing is formally zero, but other processes can still drive mixing (e.g., gravity waves), as have been studied for the Solar System planets and brown dwarfs (e.g., Bishop et al. 1995, Freytag et al. 2010).

Chemical loss timescales are more difficult to specify. As reviewed by Zahnle \& Marley (2014), one option is to determine the loss timescale by associating it with the rate-limiting step in the loss reaction (although determining this step can be difficult). Alternatively, gridded results from a large suite of photochemical models can be used to determine a functional form of $t_{\text {chem }}$. This approach was adopted by Zahnle \& Marley (2014), who propose using an Arrhenius-like rate,

$$
t_{\mathrm{chem}}=A P^{-b}[\mathrm{M} / \mathrm{H}]^{-c} e^{B / T}
$$

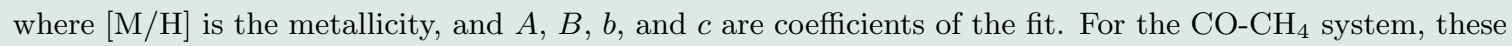
authors find

$$
t_{\text {chem }}=1.5 \times 10^{-6} P^{-1}[\mathrm{M} / \mathrm{H}]^{-0.7} e^{42000 / T} \mathrm{sec} .
$$

the incident ultraviolet flux is far higher.

\section{Gas Opacity}

The transition from hot, atomic, continuum-opacity dominated solar-like stars to the cool, mostly neutral, and molecular atmospheres of the late M, L, T, and Y dwarfs is nowhere more apparent than in the opacities which must be considered to model these atmospheres. Properly accounting for the molecular opacity of key atmospheric constituents is a major task of cool atmosphere modeling. This section reviews the important gaseous opacities in cool atmospheres, focusing on the treatment of molecular absorption lines. Techniques for solving the RTE given a complex spectrum of gas opacities are also discussed. 


\subsection{Line Strengths and Shapes}

Each molecular absorption band is formed from a collection of individual absorption lines, as shown in Figure 6, with each line marking a transition in the coupled rotational-vibrational energy state of the molecule. The shapes and strengths of these lines depend on a number of processes, including structural properties of the molecule as well as the local atmospheric conditions where the line is formed.

In general, an individual spectral line is described by three key parameters - the line position (i.e., the frequency or wavenumber at line center, $\nu_{0}$, typically in units of $\mathrm{cm}^{-1}$ ), the line strength $\left(S=S(T)\right.$, in units of $\mathrm{cm}^{2}$ molecule ${ }^{-1} \mathrm{~cm}^{-1}$, or equivalent), and the line shape function $\left(f\left(\nu-\nu_{0}\right)\right.$, in units of $\left.1 / \mathrm{cm}^{-1}\right)$. The frequency-dependent absorption coefficient for the line, $k_{\nu}$, is then expressed as

$$
k_{\nu}=S f\left(\nu-\nu_{0}\right)
$$

where the line shape function is normalized such that

$$
\int_{-\infty}^{+\infty} f\left(\nu-\nu_{0}\right) d \nu=1
$$

Thus, the integrated area under an individual line is constant (equal to $S$ ), such that broader absorption lines increase opacity in line wings at the expense of opacity near line center.

Large databases, usually referred to as line lists, compile the necessary information for computing absorption line spectra. These databases are based on either lab measurements or quantum chemistry simulations. As a number of line parameters depend on temperature, line lists are typically referenced to a standard temperature (e.g., 296 K). Commonly used line lists include the HITRAN (Rothman et al. 1987, 2013), HITEMP (Rothman et al. 1995, 2010), and ExoMol (Tennyson \& Yurchenko 2012) databases, where the latter is more appropriate for the range of temperatures encountered in sub-stellar atmospheres, but only includes data for $\mathrm{H}_{2} \mathrm{O}, \mathrm{CO}_{2}, \mathrm{CO}, \mathrm{NO}$, and $\mathrm{OH}$. Sources of line lists for more exotic species, and discussion of how to implement these databases, are reviewed and/or tabulated in several recent papers (Sharp \& Burrows 2007, Freedman et al. 2008, 2014, Lupu et al. 2014).

Given a line strength $S_{0}$, defined at a standard temperature $T_{0}$, the temperaturedependent line strength can be computed from (McClatchey et al. 1973)

$$
S(T)=S_{0} \frac{Q\left(T_{0}\right)}{Q(T)} \exp \left[\frac{E^{\prime \prime}}{k_{B}}\left(\frac{1}{T_{0}}-\frac{1}{T}\right)\right] \frac{1-\exp \left(-h c \nu_{0} / k_{B} T\right)}{1-\exp \left(-h c \nu_{0} / k_{B} T_{0}\right)},
$$

where $Q$ is the internal molecular partition function for vibrational and rotational states, $E^{\prime \prime}$ is the lower energy level for the rotational-vibrational transition (commonly supplied in line lists), $c$ is the speed of light $\left(c=2.998 \times 10^{10} \mathrm{~cm} \mathrm{~s}^{-1}\right)$, and $h$ is Planck's constant $\left(h=6.626 \times 10^{-27} \mathrm{erg} \mathrm{s}\right)$. The exponential term in the middle of this expression is the familiar Boltzmann distribution for energy states. Standard methods exist for computing partition functions (e.g., Gamache et al. 1990), which describe the temperature-dependent partitioning of rotational-vibrational energy states. The combination of the Boltzmann factor and the partition function weight the line strength by the probability of finding molecules of a given energy state within the ensemble of all states. Finally, the ratio of terms at the end of this expression corrects for 'stimulated emission,' where a molecule is deexcited from the higher energy state $\left(E^{\prime \prime}+h c \nu_{0}\right)$ via an interaction with a photon of energy 
$h c \nu_{0}$, thereby giving off two photons with the same energy as the initial photon. Viewed as negative absorption, stimulated emission makes the overall strength of the absorption line slightly smaller.

The line shape depends critically on temperature, pressure, and atmospheric composition through so-called foreign broadening. At lower pressures, where molecular collisions are relatively infrequent, line broadening is dominated by Doppler effects from thermal motions, which has a characteristic Gaussian line shape function,

$$
f_{D}\left(\nu-\nu_{0}\right)=\frac{1}{\alpha_{D}} \sqrt{\frac{\ln 2}{\pi}} \exp \left[-\frac{\ln 2\left(\nu-\nu_{0}\right)^{2}}{\alpha_{D}^{2}}\right],
$$

where $\alpha_{D}$ is the Doppler line half-width at half-maximum (HWHM), given by

$$
\alpha_{D}=\frac{\nu_{0}}{c} \sqrt{2 \ln 2 \frac{k_{B} T}{m}},
$$

where $m$ is th emolecular mass. Thus, Doppler broadening is more effective at higher temperatures and for low mass molecules, which stem from an assumed Maxwellian distribution of molecular speeds. Some atmospheric models, most notably the PHOENIX models Allard \& Hauschildt 1995; Barman et al.|2001), add a 'micro-turbulent velocity' to the thermal velocity, where the former is taken to scale with the characteristic convective velocity, $w$ (see Section 4.2) (Husser et al. 2013). Physically, this scaling represents a turbulent cascade from the macro-scale to the micro-scale, where motions can broaden individual absorption lines. These velocities are typically of order $1 \mathrm{~km} \mathrm{~s}^{-1}$ which, as an example, is comparable to the gas kinetic velocity of water vapor molecules at roughly 1,000 K. In practice, the micro-turbulent velocity has been used as a parameter to better reproduce observed spectra and to account for incomplete linelists (Kurucz 1996).

Deeper in the atmosphere, at pressure levels from which most thermal emission spectra emanate, line broadening is dominated by molecular collisional effects on emission and absorption. The line shape for so-called 'pressure broadened' lines is typically taken as a Lorentzian function,

$$
f_{L}\left(\nu-\nu_{0}\right)=\frac{1}{\pi} \frac{\alpha_{L}}{\left(\nu-\nu_{0}\right)^{2}+\alpha_{L}^{2}},
$$

where $\alpha_{L}$ is the pressured-broadened HWHM. Typically $\alpha_{L}$ is computed from a width parameter, $\gamma$, obtained from a line list, where

$$
\alpha_{L}=\gamma P\left(\frac{T_{0}}{T}\right)^{n}
$$

where $n$ is a temperature-dependence exponent, commonly supplied in line lists. The width parameter is different for self-broadening, where collisions are from molecules of the same species as the absorber, versus foreign-broadening, where other species dominate the molecular collisions. In some cases, most notably for $\mathrm{H}_{2} \mathrm{O}$ in Earth's atmosphere and $\mathrm{CO}_{2}$ in Venus' atmosphere, the Lorentzian line shape has been found to either over- or underestimate absorption in the far wings of pressure-broadened lines. In these situations, it is common to apply a line shape correction, called a ' $\chi$-factor', to best reproduce the observed data (see, e.g., Winters et al. 1964 Fukabori et al. 1986, Pollack et al. 1993 Meadows \& Crisp 1996 Mlawer et al. 2012). 
Of course, there are large portions of the atmosphere where lines are influenced by both Doppler and pressure broadening. Here, a convolution of the line shapes for these two processes is used, called the Voigt line shape, which is given by

$$
f_{V}\left(\nu-\nu_{0}\right)=\frac{\alpha_{L}}{\pi^{3 / 2}} \int_{-\infty}^{+\infty} \frac{\exp \left(-y^{2}\right)}{\left(\nu-\nu_{0}-\alpha_{D} \cdot y\right)^{2}+\alpha_{L}^{2}} d y
$$

Schreier (2011) discusses efficient methods for computing this expression. Figure 7 compares lineshapes for Doppler broadening (both with and without a micro-turbulent velocity), pressure broadening, and the combination of these into a Voigt lineshape.

An important source of uncertainty in opacity calculations is that the width parameter and the temperature-dependence exponent are usually measured for terrestrial air as the broadening gas, and are expected to have different values when other gases (e.g., $\mathrm{H}_{2}$ ) are the primary source of pressure broadening. Pressure broadening parameters for either $\mathrm{H}_{2}$ or $\mathrm{H}_{2}+\mathrm{He}$ as the background gas are available for a limited set of molecules, and do not typically span a wide range of wavelengths (Bulanin et al. 1984, Le Moal \& Severin 1986. Margolis 1993, 1996. Brown \& Plymate 1996, Gamache et al. 1996 Gabard 2013). These data are commonly extrapolated to cover the full range of wavelengths of interest. When broadening parameters are only available for terrestrial air, width parameters and temperature-dependence exponents are sometimes adjusted by a constant factor derived from insight and a limited set of available data.

Additionally, the power-law temperature dependence in the expression for the Lorentz HWHM likely does not hold over a wide range of temperatures. This is especially problematic for astrophysical applications, where the physical environment is typically quite different from the laboratory conditions where line parameters are measured. Thus theoretical modeling of line shape parameters is commonly employed, where a wide range of combinations of temperatures, pressures, and broadening gases can be investigated (Gabard 2013. Gamache \& Lamouroux 2013).

Even though most of the theory outlined above is straightforward, issues arise due to the scope of the problem. Line lists can contain more than $10^{9}$ or even $10^{10}$ transitions, which makes assembling opacities computationally expensive. Efficiency can be gained by omitting weak lines from opacity calculations, which is most effective when gas concentrations are known (or can be estimated) a priori. Additionally, it isn't feasible to sum the contributions from such large numbers of lines out to arbitrarily large distances from line centers. Thus, line shape profiles are commonly truncated at some distance from line center, which necessitates a re-scaling of the line shape to maintain the normalization of Equation 20, thus preserving the integrated line strength (Sharp \& Burrows 2007).

Finally, while the basic procedure for applying molecular opacity databases to atmospheric modeling problems can be easily outlined, there are many subtleties (for example detailed choices about line broadening, or recognizing errors, which are not uncommon, in the databases). Decision making in these cases benefits from deep experience in the construction and use of line databases. However, relatively few young astronomers specialize specifically in the study of molecular opacity issues, and this lack of new talent may represent a problem for the field in the future.

\subsection{Alkali Opacity}

At the time of the first $\mathrm{T}$ dwarf discoveries, a major shortcoming of the $\mathrm{T}$ dwarf models was that they predicted far too much flux in the far red $(\sim 900$ to $1000 \mathrm{~nm})$ compared to 
observations. One early suggested solution even relied upon a high altitude, scattering haze to account for the discrepancy (Griffith et al. 1998). In fact the missing opacity source was soon found to be the highly pressure broadened wings of resonant $\mathrm{Na}$ and $\mathrm{K}$ lines (Burrows et al. 2000).

Correctly computing the opacity of these resonant lines is not straightforward and mismatches between models and data near $1000 \mathrm{~nm}$ are commonly attributed to shortcomings in the treatment of the resonant alkali lines. Recent work by Allard et al. (2012) on these line shapes should improve the situation, but there are many subtleties in the use of the theoretical results and there is clearly room for more study of the alkali opacities.

\subsection{Continuum Gaseous Opacities}

In addition to line and molecular opacities, there are several continuum opacity sources that must be considered. Foremost among these is the collision-induced absorption (or CIA, sometimes termed pressure-induced absorption) opacity arising from transitions within supramolecules transiently created during collisions of $\mathrm{H}_{2}$ molecules with other other gas species, predominantly $\mathrm{H}_{2}$ and He. From the uncertainty principle, because collision timescales are very short, energy level transitions within the short-lived supramolecules are not sharply defined and the resulting opacity is generally smoothly varying with wavelength. A recent compendium can be found in Richard et al. (2012).

While electron densities are generally quite low in these atmospheres (Figure 3), continuum opacity sources associated with free electrons should be accounted for. These include bound-free absorption by $\mathrm{H}$ and $\mathrm{H}^{-}$and free-free absorption by $\mathrm{H}, \mathrm{H}_{2}, \mathrm{H}_{2}^{-}$, and $\mathrm{H}^{-}$(see Lenzuni et al. (1991)), as well as electron scattering.

Finally, Rayleigh scattering in the gas is also important for problems involving incident starlight. Rigorous calculations also account for Raman scattering, an important process in the UV for giant planet atmospheres (Pollack et al. 1986).

\subsection{Opacities and the RTE}

As discussed by Goody \& Yung (1989, p. 125), radiative calculations involve four distinct scales of wavelength-variation: the scale at which the Planck function varies, the scale of gas rotational-vibrational absorption bands, the scale of individual absorption lines, and, most finely, the 'monochromatic' scale at which the absorption coefficient can be considered to be constant. Techniques exist for averaging wavelength-dependent atmospheric opacities across this full range of scales, with the purpose of increasing the speed at which net radiative heating rates can be determined.

6.4.1. Gray Opacities. Early studies of the structure and evolution of brown dwarfs (Lunine et al. 1986, 1989) and recent analytic models of the thermal structure of irradiated planetary atmospheres (Hansen 2008, Guillot 2010, Robinson \& Catling 2012, Parmentier \& Guillot 2014) have used opacities averaged over the entire spectral range, or 'gray' opacities, combined with the gray two-stream equations (e.g. Andrews 2010, p. 84), to compute thermal fluxes in sub-stellar atmospheres. Typically either the Planck mean opacity, $k_{P}$, or the Rosseland mean opacity, $k_{R}$, are used, whose averaging are defined, respectively, by

$$
k_{P}(P, T)=\frac{\int_{0}^{\infty} k_{\nu}(P, T) B_{\nu}(T) d \nu}{\int_{0}^{\infty} B_{\nu}(T) d \nu},
$$




$$
k_{R}^{-1}(P, T)=\frac{\int_{0}^{\infty} k_{\nu}^{-1}(P, T) d B_{\nu}(T) / d T d \nu}{\int_{0}^{\infty} d B_{\nu}(T) / d T d \nu} .
$$

The Planck mean is weighted towards spectral regions with large opacities, yields the correct flux emitted by an atmospheric layer of temperature $T$, and is best applied in the higher, more optically thin regions of an atmosphere. Conversely, the Rosseland mean is weighted towards spectral regions of low opacity, and is designed to ensure the radiation diffusion limit in the opaque regions deep in an atmosphere (Mihalas 1970, p. 38).

Tabulating Planck and Rosseland mean opacities as a function of temperature and pressure using pre-computed, high resolution gas opacities is a difficult and tedious task. This is especially true for the Rosseland mean, whose weighting towards low-opacity spectral regions places extra emphasis on the poorly-understood far wings of spectral lines. Earlier models relied on Tsuji (1971) for gray opacities. More recently, Freedman et al. (2008, 2014) presented tables of Rosseland and Planck mean opacities appropriate for use in brown dwarf and gas giant thermal structure models.

6.4.2. Band Models. At the scale of molecular bands, numerous so-called 'band models' have been historically applied to determine thermal radiative fluxes in planetary and stellar atmospheres. While not widely used today, band models take advantage of the overall smooth nature of band-averaged transmission functions (particularly true for the relatively dense and cool brown dwarf and giant planet atmospheres), $\mathcal{T}_{\Delta \nu}$, with

$$
\mathcal{T}_{\Delta \nu}=\frac{1}{\Delta \nu} \int_{\Delta \nu} \mathcal{T}_{\nu} d \nu=\frac{1}{\Delta \nu} \int_{\Delta \nu} e^{-\left[\tau_{\nu}\left(P_{2}\right)-\tau_{\nu}\left(P_{1}\right)\right]} d \nu
$$

where $P_{1}$ and $P_{2}$ are atmospheric pressures (with $P_{2}>P_{1}$ ), and the final step uses the definition of transmissivity. The band-average transmission functions for an individual gas can be tabulated using high-resolution, wavelength-dependent opacities, and are often parameterized by a fit to a particular type of band model, such as the Godson, Malkmus, or Elsasser models (Goody \& Yung 1989, Chap. 4). The treatment of band-averaged transmission functions is more complicated in spectral regions where more than one absorbing gas have overlapping features (Pierrehumbert 2010. Sec. 4.4.5), but, nevertheless, combined transmission functions can be used to solve the two-stream thermal RTE.

A particular type of band-model, the Just Overlapping Line Approximation (JOLA), was used in early studies of brown dwarf atmospheres by Allard and collaborators (e.g., Allard \& Hauschildt 1995) and by Tsuji and collaborators (Tsuji 1984, 1994, 2002). Carbon (1979) reviews this and other types of band models.

6.4.3. Correlated- $\boldsymbol{k}$. Spectral integrals of functions that have complicated variation in wavelength (e.g., Equation 29 are computationally expensive. However, over a wavelength range where the source function and the scattering properties of the atmosphere can be taken to be roughly constant, a great deal of computational efficiency can be gained by using the distribution of opacities to replace the integral over frequency with a more well-behaved integral over a new dependent variable. This, effectively, re-orders the opacities in wavelength space, creating a smooth, monotonic description of the absorption coefficients, and is the root of the $k$-distribution approach (Ambartzumian 1936, Kondratyev 1965 Arking \& Grossman 1972, Domoto 1974).

Given the distribution function of gas opacities, $f(k)$ (where, then, the fraction of absorption coefficients between $k$ and $k+d k$ is $f(k) d k$ ), the mean transmission through an 
atmospheric layer (Equation 29 can be expressed as (Goody \& Yung 1989, Sec. 4.8),

$$
\mathcal{T}_{\Delta \nu}=\int_{0}^{\infty} f(k) e^{-k \mathcal{M}} d k
$$

where the distribution function is over the interval $\Delta \nu$, and $\mathcal{M}$ is the column mass of the layer. Since the distribution function is a relatively smooth function of $k$, the integral in Equation 30 can be computed with much less computational effort than that in Equation 29. The integral in Equation 30 can be made even more straightforward if we replace the distribution function with the cumulative distribution function, $g(k)$, with

$$
g(k)=\int_{0}^{k} f\left(k^{\prime}\right) d k^{\prime},
$$

so that the mean transmission is then

$$
\mathcal{T}_{\Delta \nu}=\int_{0}^{1} e^{-k_{g} \mathcal{M}} d g
$$

where $k_{g}$ indicates the mapping between $k$ and $g$ (i.e., $k_{g}$ is the value of $k$ that corresponds to the independent variable $g$ ). Given the smooth, monotonic nature of $g(k)$, this integral can be accurately evaluated using only 10-20 intervals in $g$ or less (Goody et al. 1989).

Instead of determining the transmission over $\Delta \nu$ using Equation 32 and then solving the RTE, which would not allow for the treatment of scattering, many $k$-distribution models will, instead, use the $k-g$ mapping to determine characteristic absorption coefficients for ranges of $g$ (Yamamoto et al. 1970, Lacis \& Hansen 1974, Liou 1974, Ackerman et al. 1976. Mlawer et al. 1997), called $k$-coefficients. Along with the relevant thermal and scattering source terms (assumed constant over $\Delta \nu$ ), the $k$-coefficients are then used to solve the RTE. The fluxes from these calculations are then combined based on the width of the range of $g$ values used, effectively swapping the order of integration over frequency and with solving the RTE. As opacities depend on temperature, pressure, and gas composition, the $k$-coefficients must be computed for each model atmospheric layer, or, most commonly, obtained from pre-tabulated results.

In practice, two key complications arise when using $k$-distributions to perform radiative transfer calculations in inhomogeneous atmospheres. First, the particular ordering of opacities in wavelength space that maps to $g(k)$ for a particular atmospheric level need not be the same ordering as at any other level in the model. If the absorption coefficients are indeed spectrally uncorrelated with those at another level, then different wavelengths are being mixed when solving the RTE for the entire atmosphere. Thus, it is assumed, as is often the case, that absorption coefficients throughout the atmosphere are spectrally correlated, leading to the so-called correlated- $k$ method (Lacis \& Oinas 1991). In practice this limitation can be overcome by using narrow spectral intervals and choosing interval boundaries so that one main absorber dominates each interval.

Second, the blending of $k$-coefficients, computed for an individual gaseous species, with those for other species to represent a realistic mixture of gases in an atmosphere remains controversial. Most correctly, the $k$-coefficients should be computed for a mixture of gases, but it is often the case in atmospheric modeling that gas mixing ratios are not known a priori (which happens when, e.g., the concentration of a gas depends on other atmospheric state variables). Thus, a more flexible approach is to compute the $k$-coefficients for each individual gas, and then blend these 'on the fly' in model runs. However, as discussed by 
Goody et al. (1989), this approach is only valid when the absorption coefficients for the individual gases are spectrally uncorrelated. Furthermore, blending $k$-coefficients for gas mixtures can be computationally expensive, as a spectral interval with $n$ different absorbers requires calculation $n^{n}$ permutations of $k$-coefficients. Because of the uncertain magnitude of the error introduced by blending single-gas k-coefficients, this approach should only be used with some care.

Complications aside, correlated- $k$ techniques have proven to be efficient and accurate. Extensive testing in the Earth science literature has shown that correlated- $k$ methods can achieve flux and heating rate accuracies to within 1-5\%, when compared to more precise techniques (Lacis \& Oinas 1991, Fu \& Liou 1992, Mlawer et al. 1997). Goody et al. (1989) note occasional cases where errors are much larger than several percent, and caution that expanding the use of correlated- $k$ techniques into new applications (i.e., beyond the Earth sciences) requires testing against more reliable methods to ensure the validity of the approach. Internal tests by the Marley/Saumon group have found that, for cloud-free models, the correlated- $k$ approach is accurate to within about $1 \%$ for brown dwarfs when compared to rigorous line-by-line calculations - a level of accuracy that exceeds most other uncertainties in the problem.

6.4.4. Line-by-Line. At spectral scales where the radiative source terms and all opacity sources can be considered to be constant (i.e., at resolutions typically less than about $0.01 \mathrm{~cm}^{-1}$ ), radiative transfer calculations are monochromatic, and computing spectrallyaveraged opacities is no longer an issue. Models that solve the monochromatic RTE over a fine grid of wavelengths are referred to as 'line-by-line' radiative transfer models. This technique is considered to be the most accurate method for dealing with complex opacities, but the 'brute force' nature of the approach makes the technique computationally expensive. Line-by-line methods can be made more efficient through the use of spectral mapping techniques (West et al. 1990 Meadows \& Crisp 1996), where monochromatic elements with similar optical properties at all atmospheric levels are binned together, with the RTE being solved only once for the bin instead of for every monochromatic element.

A sample comparison between a line-by-line model spectrum $\left(T_{\text {eff }}=1900 \mathrm{~K}\right)$ and data for an L2 dwarf at a spectral resolution of $\lambda / \Delta \lambda=50,000$ is shown in Figure 8 This spectral resolution, which is much higher than typical brown dwarf spectra, is sufficient to resolve individual lines. While the model (from Marley et al. 2002) has been convolved with a rotational kernel, the overall agreement in this particular case demonstrates that the model line broadening treatment generally reproduces the data. Note that this particular model employed assumes no micro-turbulent broadening.

Finally, 'direct opacity sampling' (dOS) (Hauschildt et al. 2001), like line-by-line calculations, solves the RTE monochromatically, and is the method for handling opacities in the RTE currently used in the PHOENIX group of models (Allard \& Hauschildt 1995, Barman et al. 2001). Note that dOS is distinguished from standard opacity sampling methods, which use statistical techniques (e.g., weighting by a Planck function) to sample the wavelengthdependent opacity distribution, in that dOS uses a pre-specified spectral grid, which can be set to very fine resolutions. In practice, computational cost limits dOS calculations to $\sim 1 \mathrm{~cm}^{-1}$, which is sufficient to accurately compute the radiative fluxes, but is not as fine as true line-by-line calculations. 


\section{Cloud Opacity}

The problem of modeling, to some degree of fidelity, the clouds in ultracool dwarfs and giant planets is a central challenge to understanding the atmospheric structure, reflected and emitted spectra, and even evolution of these objects. The subject is complex and there are many different approaches. In this section we give an overview of the problem and some perspective on the various modeling approaches. A more detailed review can be found in Marley et al. (2013), and detailed comparisons of cloud treatments can be found in Helling et al. (2008c) (which is now slightly dated).

\subsection{Condensates Overview}

As in the cool, dense molecular atmospheres found within the Solar System, a variety of species condense within the atmospheres of brown dwarfs. The resulting clouds present the single greatest obstacle impeding our understanding of these objects. To correctly account for cloud opacity, it is necessary to model each condensate-forming species, estimating particle sizes and vertical distributions. Predicting whether condensates are sub-micron in size and are distributed vertically throughout the atmosphere, to millibar pressures for example, or whether particles are large and mostly confined to thin cloud decks, or are something in between, is the central task of condensate modeling. The difficulty in accomplishing this, and of reconciling diverse modeling approaches with data, limits progress and understanding. While a number of innovative and insightful cloud models have been developed to facilitate modeling of brown dwarf atmospheres and the interpretation of data, there has been remarkably little progress in the past decade and the problem of cloud opacity provides the greatest opportunity to realize improvements in model atmosphere fidelity.

At the relatively cool atmospheric temperatures of brown dwarfs and giant planets, important atmospheric constituents are expected to be found in condensed phases, particularly $\mathrm{Fe}, \mathrm{Si}$, and $\mathrm{Mg}$, but also the more refractory components, including $\mathrm{Al}, \mathrm{Ca}, \mathrm{Ti}$, and $\mathrm{V}$. Table 1 lists many of the important condensates predicted by rainout chemical equilibrium.

For the case of homogeneous condensation, where the gas phase species condenses to form a solid or liquid of the same species (e.g., $\mathrm{Fe}$ or $\mathrm{H}_{2} \mathrm{O}$ ), condensation first occurs in a rising parcel of gas when the local partial pressure of the condensing gas first exceeds the saturation vapor pressure. This defines the cloud base. Supersaturation is a measure of how far in excess of the saturation vapor pressure the gas must be in order to condense. In more complex cases (e.g., $\mathrm{Ca}$ and $\mathrm{TiO}$ forming $\mathrm{CaTiO}_{3}$ ) the cloud base is determined in principle through chemical equilibrium calculations. Figure 9 shows the condensation boundaries, again for rainout chemistry, for many key species along with a collection of model pressure-temperature profiles for reference. The marked boundaries in the figure are the locations where the labeled solid or liquid species will form as its progenitor gaseous species are carried upwards in a rising air parcel.

As Figure 9 attests, there are numerous atmospheric condensates within the brown dwarf pressure-temperature regime. However not all of them are equally important. Some species, such as TiO, play a leading role in controlling gaseous opacity in the $\mathrm{M}$ and early L dwarfs, but because of their low abundance, are relatively unimportant cloud opacity sources. The more abundant Fe-, Si-, and Mg-bearing species have a greater contribution to column grain optical depths. The role of elemental abundances and particle sizes can best be appreciated by constructing a highly simplistic cloud model (Marley 2000), a task that also illustrates the challenge faced by cloud modelers. 
If we assume that all molecules of a species with a fractional number mixing ratio, $f$, resident above a given pressure level, $P_{\mathrm{c}}$, condense, then, for a hydrostatic atmosphere, the column mass of the condensate, $\mathcal{M}_{\mathrm{c}}$, is given by

$$
\mathcal{M}_{\mathrm{c}}=f\left(\frac{m_{\mathrm{c}}}{m}\right)\left(\frac{P_{\mathrm{c}}}{g}\right)
$$

where $m_{\mathrm{c}}$ is the molecular mass of the condensed species and $m$ is the mean molecular mass of the atmosphere. Table 2 gives $\varphi=f m_{\mathrm{c}} / m$ and the condensation temperature at 3 bar in a solar composition gas for several important species. Given this column mass, the total condensate optical depth as a function of wavelength, $\lambda$, can be estimated given the particle size, $r_{\mathrm{c}}$, the extinction efficiency, $Q_{\lambda}^{\text {ext }}\left(r_{\mathrm{c}}\right)$ (which can be derived from Mie theory), and the condensate mass density, $\rho_{\mathrm{c}}$,

$$
\tau_{\lambda}=75 \epsilon Q_{\lambda}^{\mathrm{ext}}\left(r_{\mathrm{c}}\right) \varphi\left(\frac{P_{\mathrm{c}}}{1 \mathrm{bar}}\right)\left(\frac{10^{5} \mathrm{~cm} \mathrm{~s}^{-2}}{g}\right)\left(\frac{1 \mu \mathrm{m}}{r_{\mathrm{c}}}\right)\left(\frac{1 \mathrm{~g} \mathrm{~cm}^{-3}}{\rho_{\mathrm{c}}}\right) .
$$

In a real atmosphere, not all of the condensible species is found in the condensed phase, so this parametrization introduces a term, $\epsilon<1$, to account for such effects.

Equation 34 exemplifies many of the challenges presented by atmospheric condensates. While we can estimate the column mass of a given material that may be found in the condensed phase, the actual opacity depends sensitively upon the particle size, both through the mass partition term and the Mie extinction. Figure 10 shows spectra of Mie absorption and scattering efficiencies, where $Q_{\lambda}^{\text {ext }}=Q_{\lambda}^{\text {abs }}+Q_{\lambda}^{\text {scat }}$, for a collection of condensates and for several different particle sizes. Furthermore, the mass balance in Equation 34 tells us nothing about the vertical distribution of the grains above a condensation level. An additional limitation of course is that basic Mie theory (for a modern review of the history of the method and summary of available codes, see Wriedt 2012) assumes that the condensates are ideal, spherical particles.

All else being equal, however, reference to Table 2 demonstrates that knowing the fractional abundance of a condensate is crucial to understanding its potential contribution to cloud opacity. The single most important condensate over the range of temperatures expected for brown dwarf atmospheres is $\mathrm{H}_{2} \mathrm{O}$. Iron and silicate phases are the second most important species, while the refractory oxides, such as $\mathrm{CaTiO}_{3}$, and the alkali species, such as $\mathrm{KCl}$, are of tertiary importance.

\subsection{Modeling Approaches}

One could begin to compute the vertical distribution of cloud opacity by considering several possible fates for atmospheric grains after they have formed. In one extreme, the grains might immediately fall out of the atmosphere, leaving behind a clean gas. Fortuitously, the first indisputable brown dwarf, Gliese 229B, had such an atmosphere, and models that entirely neglected grain opacity did a reasonable job of reproducing the observed spectra. Many groups today continue to produce cloud-free models, although they differ in the details of how the chemistry is treated. Examples include the COND models of the PHOENIX group, and various model formulations from other groups including the cloudless models of Saumon \& Marley (2008). At the other extreme, grains might stay in chemical equilibrium with the local gas and not precipitate at all. This is the domain of the DUSTY models of Allard et al. (2001). Recall that these two extremes also describe treatments of rainout 
chemistry discussed in Section 5.1.2. All other cloud models attempt to define some intermediate case where grains grow and fall from the upper reaches of the atmosphere, but do not completely disperse, thus forming cloud decks of some finite vertical extent.

One approach attempts to empirically describe clouds by varying only a few parameters. Typically this is a cloud bottom, at the condensation level, and top pressure (or, equivalently, a bottom pressure and fractional vertical scale height) and a particle size. Examples of such 'defined cloud' models include the 'unified' models of Tsuji et al. (Tsuji 2002) and various cloud models employed by Burrows and collaborators (Burrows et al. 2002; Currie et al. 2011). The strength of this approach is that no cloud physics need be crafted, but rather a set of empirical cloud descriptions can be accumulated as progressively more objects are compared to models. In principle, more sophisticated models could be constructed that attempt to explain trends in particle sizes or cloud thicknesses once a sufficient number of objects have been characterized. In practice, however, this has not yet happened and we are left with individualized descriptions of particular objects. This approach also has little predictive value, as it is not obvious what parameters might be appropriate to describe new objects.

Nevertheless, this empirical method has contributed to the definitive finding that neither the cloudless nor fully dusty concepts are correct for the L dwarfs-brown dwarf clouds are found in discrete layers, as are clouds throughout the Solar System. Indeed, this limitation of the DUSTY modeling approach is an important one. Since the DUSTY models assume that condensates do not form cloud layers, for cooler models the atmospheric column becomes optically thick very rapidly, even when just accounting for the grain opacity. By $T_{\text {eff }} \sim$ $1300 \mathrm{~K}$, DUSTY models are far redder, with far shallower molecular bands, than even the cloudiest known L dwarfs. For this reason, fits of DUSTY models to data seldom find effective temperatures below 1400 or even $1500 \mathrm{~K}$.

One example of a model that attempts to capture some aspects of cloud physics to predict particle sizes and vertical distributions is the Eddysed model developed by Ackerman \& Marley (2001). This model balances upwards and downwards transport of condensible gases and condensates by way of a tunable 'sedimentation efficiency' parameter, $f_{\text {sed }}$. As $f_{\text {sed }}$ increases, downwards mass transport by falling particles becomes more efficient and the clouds become thinner. The code computes the particle sizes that are required to produce the implied mass balance rather than attempting to model grain growth explicitly.

A unique cloud modeling paradigm has been developed by Helling et al. in a series of papers (e.g., Helling et al. 2001, Helling \& Woitke 2006, Helling et al. 2008a b and references therein). The key underlying assumption of this approach is that-not unlike the conversion of $\mathrm{CO}$ to $\mathrm{CH}_{4}$ - condensation is difficult; the formation of many of the condensates predicted by chemical equilibrium requires the collisions and reactions of multiple gaseous components (e.g., $\mathrm{Ca}$ and $\mathrm{TiO}$ molecules, both of which are relatively rare in the predominantly hydrogen-helium gas). This approach thus posits that an equilibrium progression of distinct cloud layers, as predicted by equilibrium chemistry, is unlikely. Instead this paradigm follows the growth of condensate 'seed particles' that fall down from the top of the atmosphere, facilitating the nucleation of a sequence of compounds that are formed during the downward drift of particles from above. Instead of the canonical equilibrium cloud layers, this approach predicts a blend of 'dirty' grains, with a complex, mixed composition that varies continuously with height.

Helling and collaborators have explored the various ramifications of this approach in greater detail than can be summarized here (recently in Witte et al. (2011)). The main 
takeaway, however, is that the canonical condensation sequence of Figure 9 is an overly idealized conception of reality. However, to reach this conclusion, the Helling et al. model posits what may be an equally unlikely scenario. Here, the highly refractory 'seed particles' are taken to be carried by strong updrafts from the deep atmosphere to the top of the atmosphere, where they begin their downward journey. These $\mathrm{TiO}_{2}$ particles are presumed to be transported upwards rapidly enough such that no condensation takes place onto these nuclei and that none of the other condensible species that are also entrained in the updraft condense during the upward journey. In the limit of cold Solar System giant planets, we know that this is not the case as there is no such high altitude refractory haze observed. Another practical difficulty with this model is that the rich brew of condensates imprints a complex signature on models of emergent spectra, such that it is challenging to appreciate the effect of any single component of the model.

Despite the aforementioned concerns, there is much to recommend the Helling et al viewpoint, and only detailed comparisons of model predictions to data will ascertain the validity of this model conception. Indeed the 'Drift-PHOENIX' set of forward models has adapted a version of the Helling et al models, although as of the time of preparation of this review, the models have not been described in the literature.

\section{Deriving the Thermal Profile}

In practice, a one-dimensional radiative-convective thermal structure profile must be derived iteratively, starting from a first guess. There are several approaches that can be followed to determine the equilibrium profile, which satisfies Equation 4, and where the temperature and flux profiles are all continuous. A straightforward technique is to simply timestep the atmosphere to equilibrium, which dates back to some of the earliest radiative-convective models (Manabe \& Strickler 1964). However, more efficient computational approaches to determining the equilibrium thermal structure have been developed, and, here, simply as a means of bringing together the previous sections, we sketch out one technique that has been successfully applied to Solar System, extrasolar planet, and brown dwarf atmospheres (McKay et al. 1989, Marley et al. 1996, Marley \& McKay 1999, Fortney et al. 2005).

We begin with an atmosphere grid that has some number, $N$, of discrete levels, denoted by $i$ (Figure 1). The topmost level is taken as $i=1$, and each level is associated with a fixed pressure. If the radiative-convective boundary falls at level $i_{\mathrm{RC}}$, then, as was discussed in Section 4.1, the temperature at each point in the convection zone (i.e., the points where $N \geq$ $\left.i \geq i_{\mathrm{RC}}\right)$ can be found by following an adiabatic gradient downwards from the temperature at the radiative-convective boundary, $T_{\mathrm{RC}}$. Thus, our task is to solve for the temperature at each level where $i<i_{\mathrm{RC}}$. Here, the atmosphere is in radiative equilibrium, and, for non-irradiated bodies, the net thermal flux through each level must be (from Equation 4 )

$$
F_{i, \text { target }}^{\text {net }}=\sigma T_{\mathrm{eff}}^{4},
$$

where $T_{\text {eff }}$ is the desired effective temperature for the model, and the thermal subscript has been dropped for clarity. For irradiated planets, the net thermal flux must be increased by the net stellar flux at each level.

For a first guess atmosphere profile $T(P)$, chemical abundances must be determined (Section 5 and distributions of any relevant aerosols must be found (Section 7). From these, the wavelength-dependent opacities can be computed (or obtained from a look-up table; Section 6), thereby permitting the calculation of the net thermal flux profile (Sec- 
tion 3. In general, though, the net thermal fluxes at the model levels throughout the radiative zone will not sum to the target value, implying a net radiative heating or cooling (Equation 5). From the mismatch with the target, a flux error can be computed, and is given by $\delta F_{i}^{\text {net }}=F_{i}^{\text {net }}-F_{i, \text { target }}^{\text {net }}$ The task of the model is then to correct the first guess $T(P)$ profile to find an improved guess. This is a complex problem, as the flux at each level in the atmosphere depends on the temperature structure of all other layers. So $F_{i}^{\text {net }}=F_{i}^{\text {net }}\left(T_{1}, T_{2}, T_{3}, \ldots, T_{\mathrm{RC}}\right)$. Note that, since $T_{\mathrm{RC}}$ controls the temperature profile down the adiabatic gradient through the convection zone, $F_{i}^{\text {net }}$ does not explicitly depend on the temperature at levels with $i>i_{\mathrm{RC}}$, as these temperatures are uniquely specified by $T_{\mathrm{RC}}$ and the adiabat. A Jacobian matrix, $\overleftrightarrow{\boldsymbol{A}}$, of partial derivatives describes how the flux at each level in the atmosphere depends upon the temperature at each level,

$$
\overleftrightarrow{\boldsymbol{A}}=\left(\begin{array}{cccc}
\frac{\partial F_{1}}{\partial T_{1}} & \frac{\partial F_{1}}{\partial T_{2}} & \cdots & \frac{\partial F_{1}}{\partial T_{\mathrm{RC}}} \\
\frac{\partial F_{2}}{\partial T_{1}} & \frac{\partial F_{2}}{\partial T_{2}} & \cdots & \frac{\partial F_{2}}{\partial T_{\mathrm{RC}}} \\
\vdots & \vdots & \ddots & \vdots \\
\frac{\partial F_{\mathrm{RC}}}{\partial T_{1}} & \frac{\partial F_{\mathrm{RC}}}{\partial T_{2}} & \cdots & \frac{\partial F_{\mathrm{RC}}}{\partial T_{\mathrm{RC}}}
\end{array}\right)
$$

The individual terms in the Jacobian matrix are computed by iteratively perturbing the temperature at each level in the model and re-computing the net fluxes throughout the atmosphere. This is a time-consuming step as the entire radiative transfer must be solved $i_{\mathrm{RC}}$ times, each occasion with a temperature perturbation introduced at a single level. Once $\overleftrightarrow{\boldsymbol{A}}$ is in hand it must be inverted to find the vector,

$$
\delta \boldsymbol{T}=\boldsymbol{A}^{-1} \cdot \delta \boldsymbol{F}
$$

where $\delta \boldsymbol{F}$ is the vector of flux errors. Then $T_{i}^{\prime}=T_{i}+\delta T_{i}$ would ideally bring all of the net thermal fluxes to the desired value (i.e., Equation 35 .

Once a temperature correction is applied and a new thermal structure is in hand, the process will repeat. In practice, the new model will not be precisely at $F_{\text {target }}^{\text {net }}$, because the problem is not linear and molecular abundances, opacities, and cloud structure will all respond to the new profile, further perturbing the computed fluxes. Thus, many iterations are required until the net thermal flux change from one iteration to the next is smaller than some small target value throughout the atmosphere, typically taken as $10^{-5}-10^{-6}$. In addition, if the temperature gradient in the bottom-most radiative layer exceeds the adiabatic lapse rate, then the lapse rate in that layer must be reset to the adiabatic value, and the radiative-convective boundary moved up to level $i_{\mathrm{RC}}-1$. In practice there are many more subtleties that arise, but, nevertheless, approaches such as this are robust and eventually converge on a desired solution.

The computational intensity of the process outlined above, particularly the repeated calculation of $\overleftrightarrow{\boldsymbol{A}}$ and the attendant radiative transfer calculations, is the reason why rapid methods for computing the radiative transfer are required for atmospheric modeling (e.g., Section 6.4). Additionally, various methods for streamlining this process and minimizing the number of matrix inversions that must be performed have been devised.

The greatest impediment to model convergence is, of course, clouds. If an iterative step cools the overall profile, then the cloud base for a photospheric condensate will move down to thicker atmospheric levels and, as a result, the cloud optical depth will increase. However, a thicker cloud will trap more thermal radiation in the atmosphere (i.e, provide a stronger greenhouse effect), which will, in turn, tend to heat the atmosphere and warm 
the temperature profile. This, then, leads to a higher cloud base, a thinner cloud, and greater cooling. Without some numerical approach to smooth convergence, it is not unusual to see model profiles vacillate between different cloud states while never converging to a mean solution. In modern terrestrial cloud modeling, in fact, clouds are never treated in one dimension because of this problem. Approaches that have been tried in brown dwarf atmospheric modeling include using trailing averages of past cloud states to smooth out jumps in the cloud state, and using two atmospheric columns, one cloudy and one clear, to allow for horizontal patchiness and reduced sensitivity to opacity changes. Details of such numerical 'tricks' are not always well described in the literature.

\section{Results}

Several research groups actively construct radiative-convective equilibrium atmosphere models, compute emergent spectra, and compare the results to data. Table 3 lists a few of the most active collaborations, and selected theory and model-data comparison papers. These papers serve as jumping off points to further explore the approaches employed by and the science results of each group.

Figure 11 present some example comparisons between models computed by the Marley/Saumon group and spectral data for a broad range of L and T dwarfs. Details of the models and data are presented in Stephens et al. (2009). The individual best fitting models were selected from a large grid of forward models computed for this purpose. As the figure attests, the quality of the matches varies from object to object. Overall the fits for the $\mathrm{T}$ dwarfs are generally quite good. Notably for the early (T0 to T2) and the one T5.5 dwarfs, thin clouds ( $f_{\text {sed }} \sim 3$ to 4 ) are favored while for the T4 dwarfs cloudless models fit best.

In contrast, for the $\mathrm{L}$ dwarfs, generally thicker clouds $\left(f_{\text {sed }}\right.$ mostly in the range of 1 to 2) are required. Cloudless models (not shown) fit far more poorly, thus demonstrating that even though there are no 'smoking-gun' spectral features indicating that clouds are present, their overall impact on the spectra are undeniable (the absence of $\mathrm{TiO}$ and other spectral features in the spectra of $\mathrm{L}$ dwarfs that are found in $\mathrm{M}$ dwarfs indicates that these species have condensed, but not necessarily into discrete cloud decks). Figure 11 also demonstrates that while the cloudy models generally reproduce the spectral shapes of each object, there are important mismatches between models and data. Since we know from the comparisons with the cloudless $\mathrm{T}$ dwarfs that the atmospheric chemistry is reasonably well understood, these mismatches point to shortcomings in the cloud model. Understanding the sources of these mismatches and how the cloud description should be modified in each case represents an important task for future research.

Witte et al. (2011) fit many of the same dwarfs as Stephens et al. (2009), and this provides an opportunity to compare derived parameters between two groups. For 2MASS J1507, an L5.5 dwarf for example, the best fitting model of Stephens et al. (2009) has $g=3 \times 10^{3} \mathrm{~m} \mathrm{~s}^{-2}$ and $T_{\text {eff }}=1600 \mathrm{~K}$. Witte et al., using the cloud model approach of Helling's group (but fitting only to the 1.0 to $3.0 \mu \mathrm{m}$ data), find $g=1 \times 10^{3} \mathrm{~m} \mathrm{~s}^{-2}$ and $T_{\text {eff }}=1800 \mathrm{~K}$. This discrepancy is almost certainly attributable to the differing cloud models and again points to the need for higher fidelity models or a new approach. 


\section{Current Issues}

\subsection{Variability and Patchy Clouds}

The emergent spectra of many L and $\mathrm{T}$ dwarfs are known to vary with time (e.g., Tinney \& Tolley 1999 Bailer-Jones \& Mundt 1999; Gelino et al. 2002, Artigau et al. 2009, Heinze et al. 2013). Broadband observations have revealed periodic and non-periodic flux variations as large as 10-30\% (Radigan et al. |2012, Gillon et al. 2013), occurring on timescales from 1-100 hr (Bailer-Jones \& Mundt 2001), and spectroscopic observations have shown that variability can be strongly wavelength-dependent (Buenzli et al. 2012$)$. While a variety of dynamical processes can influence brown dwarf spectra (Robinson \& Marley 2014 Zhang \& Showman 2014), it is generally expected that clouds play an important, if not central, role in these brightness variations, as cloud structures provide a continuum opacity source that sculpts the emergent spectra of nearly all spectral classes of brown dwarfs. Indeed, a variety of patchy cloud models have demonstrated that, in many cases, observed variability can be explained by changes in cloud distribution and thickness (Marley et al. 2010, Apai et al. 2013 Burgasser et al. 2014, Morley et al. 2014a). Furthermore, a recent report of a spatially-resolved map of a nearby L-T transition dwarf (Crossfield et al. 2014) revealed a patchy photosphere consistent with complex cloud structures.

Clearly observations of brown dwarf variability present an important opportunity for constraining cloud models and dynamical simulations. For example, Zhang \& Showman (2014) used a simple, cloud-free dynamical model to study how different circulation regimes in brown dwarf atmospheres could influence broadband lightcurves. However, realistically simulating the influence of patchy, time-evolving clouds on the emergent spectra of brown dwarfs presents a great modeling challenge. The study of brown dwarf atmospheric circulation is only just beginning (e.g., Showman \& Kaspi 2013), and, as a result, these models have not yet incorporated chemistry, aerosols, or wavelength-dependent radiative transfer. Meanwhile, one-dimensional brown dwarf atmospheric models, which do incorporate chemistry, clouds, and realistic radiative transfer, are typically only used to study an atmosphere in its steady-state. A combination of these two approaches will be needed if we are to understand the true nature and complexity of brown dwarf atmospheres.

\subsection{Atmospheric Retrieval}

The actual atmospheric state of a world, including the thermal structure and gas concentrations, can be constrained by a group of techniques called 'retrieval.' Atmospheric retrieval techniques, somtimes referred to as 'inverse methods', have their origins in the Earth remote sensing literature (Rodgers 1976, 2000), and operate by extracting information about atmospheric conditions from emitted-, reflected-, or transmitted-light spectra. From the perspective of radiative-convective modeling, the utility of retrieval is obvious - these techniques provide a direct and independent means of constraining the parameters and physics of atmospheric thermal structure models.

Hot Jupiters have seen extensive applications of retrieval techniques. Madhusudhan \& Seager (2009), by comparing observations of HD 189733b and HD 209458b to a multidimensional grid of models, reported constraints on the atmospheric thermal structure and concentrations of several key gases for these worlds. More sophisticated retrieval methods have also been applied to observations of hot Jupiters, including optimal estimation techniques (Lee et al. 2012, Line et al. 2012) and Markov chain Monte Carlo methods (Madhusudhan et al. 2011, Benneke \& Seager 2012, Line et al. 2013). Care must be taken 
when interpreting retrieval results, especially when constrained only by photometric data, as the results hinge on the assumptions incorporated into the forward model, including the particular set of gases included.

Historically, evolutionary and atmospheric processes of brown dwarfs have been constrained by comparing observed spectra of individual objects to a large grid of model spectra (e.g., Burrows et al. 1993; Burgasser et al. 2007). Uncertainties in key parameters (e.g., effective temperature) are sometimes estimated within the grid-based model comparison approach (Cushing et al. 2008; Rice et al. 2010). A drawback of this approach is that it imposes model assumptions onto the resulting fits, and the fitting approach amounts to hunting for the model parameters that best reproduce an observed spectrum

Very recently, optimal estimation retrieval techniques have been applied to hot, young directly-imaged gas giant planets (Lee et al. 2013) and brown dwarfs (Line et al. 2014). Figure 12 compares thermal profiles from a grid-based approach (Saumon et al. 2006) and an optimal estimation retrieval approach (Line et al.|2014), as applied to Gl 570D (Burgasser et al. 2000). The agreement between the two methods is quite good, except in the upper atmosphere, where the optimal estimation approach indicates a warmer thermal structure. This result hints that important physics may be missing from the models used in the gridbased approach, such as non-local thermodynamic equilibrium processes (Sorahana et al. 2014).

\section{Conclusion}

Since the discovery of the first unmistakable brown dwarf in 1995 there have been well over 3,000 papers published on this topic alone. Almost without exception, these papers, which generally aim to understand the spectra, formation, and evolution of brown dwarfs, relate in one way or another to the atmospheres of these objects. While the numbers of papers about the directly-imaged planets are-for now-smaller, there is little doubt that this field is likewise on the verge of a rapid expansion.

The science yield from these new exoplanet discoveries, as well as from ongoing studies of brown dwarfs, hinges in large part in our ability to model and understand the atmospheres of these worlds. While the first two decades of brown dwarf science has seen remarkable advances in the fidelity of atmosphere modeling, there is still much room for improvement. Better cloud models, greater exploration of the effect of varying elemental abundances, particularly atmospheric $\mathrm{C} / \mathrm{O}$ ratios, and greater studies of departures from equilibrium chemistry are all important areas for improvement. Ultimately, retrieval methods for determining atmospheric thermal, cloud, and chemical profiles likely offer the best avenue for truly constraining the properties of these objects. Retrieval methods, however, require high fidelity data - ideally taken over a large wavelength range - and this may be difficult for directly-imaged exoplanets in the foreseeable future.

Nevertheless, the convergence of modeling approaches derived from the studies of Earth, Solar System planets, exoplanets, and, of course, stellar atmospheres will continue to enrich this field. Brown dwarfs are often explained as being 'failed stars', but there is little doubt that the study of their complex, fascinating atmospheres has been one of the great successes of theoretical astrophysics over the past two decades. 


\section{Acknowledgements}

MSM acknowledges support of the NASA Planetary Atmospheres, Astrophysics Theory, and Origins programs. TDR gratefully acknowledges support from an appointment to the NASA Postdoctoral Program at NASA Ames Research Center, administered by Oak Ridge Affiliated Universities. Helpful feedback on earlier versions of this manuscript was provided by Jonathan Fortney, Didier Saumon, and David Catling. We thank Roxana Lupu for sharing results from high-resolution opacity calculations, Richard Freedman for interesting discussions regarding line shapes and strengths, Caroline Morley for sharing thermal structure models and condensation curves, Michael Line for providing output from retrieval models, Sandy Leggett, Cullen Blake, and Mike Cushing for sharing data on short notice, Kevin Zahnle and Bruce Fegley for discussions about chemistry, and many other colleagues for generously offering their insights and comments.

\section{LITERATURE CITED}

Ackerman, AS, Marley, MS. 2001. Astrophys. J. 556:872

Ackerman, TP, Liou, KN, Leovy, CB. 1976. J. Appl. Meteorol. 15:28

Allard, F, Hauschildt, PH. 1995. Astrophys. J. 445:433

Allard, F, Hauschildt, PH, Alexander, DR, Starrfield, S. 1997. Annu. Rev. Astron. Astrophys. 35:137

Allard, F, Hauschildt, PH, Alexander, DR, Tamanai, A, Schweitzer, A. 2001. Astrophys. J. 556:357

Allard, F, Hauschildt, PH, Baraffe, I, Chabrier, G. 1996. Astrophys. J. Lett. 465:L123

Allard, NF, Kielkopf, JF, Spiegelman, F, Tinetti, G, Beaulieu, JP. 2012. Astron. Astrophys. 543:A159

Allende Prieto, C, Lambert, DL, Asplund, M. 2001. Astrophys. J. Lett. 556:L63

-. 2002. Astrophys. J. Lett. 573:L137

Ambartzumian, V. 1936. Publ. Obs. Astron. Univ. Leningrad 6:7

Anders, E, Grevesse, N. 1989. Geochem. Cosmochim. Ac. 53:197

Andrews, DG. 2010. An Introduction to Atmospheric Physics. Cambridge: Cambridge Univ. Press

Apai, D, Radigan, J, Buenzli, E, et al. 2013. Astrophys. J. 768:121

Arking, A, Grossman, K. 1972. J. Atmos. Sci. 29:937

Artigau, É, Bouchard, S, Doyon, R, Lafrenière, D. 2009. Astrophys. J. 701:1534

Asplund, M, Grevesse, N, Sauval, AJ, Scott, P. 2009. Annu. Rev. Astron. Astrophys. 47:481

Bailer-Jones, CAL, Mundt, R. 1999. Astron. Astrophys. 348:800

-. 2001. Astron. Astrophys. 367:218

Baraffe, I, Chabrier, G, Allard, F, Hauschildt, PH. 2002. Astron. Astrophys. 382:563

Barman, TS, Hauschildt, PH, Allard, F. 2001. Astrophys. J. 556:885

Barman, TS, Macintosh, B, Konopacky, QM, Marois, C. 2011. Astrophys. J. 733:65

Barshay, SS, Lewis, JS. 1978. Icarus 33:593

Benneke, B, Seager, S. 2012. Astrophys. J. 753:100

Beuzit, JL, Feldt, M, Dohlen, K, et al. 2008. Soc. Photo-Opt. Instru. Eng. (SPIE) 7014

Bézard, B, Lellouch, E, Strobel, D, Maillard, JP, Drossart, P. 2002. Icarus 159:95

Bishop, J, Atreya, SK, Romani, PN, et al. 1995. In Neptune and Triton, ed. DP Cruikshank, MS Matthews, AM Schumann. Tucson: Univ. Arizona Press. 427

Blake, CH, Charbonneau, D, White, RJ, Marley, MS, Saumon, D. 2007. Astrophys. J. 666:1198

Böhm-Vitense, E. 1958. Zeitschrift für Astrophysik 46:108

Brown, LR, Plymate, C. 1996. J. Quant. Spectrosc. Radiat. Transf. 56:263

Buenzli, E, Apai, D, Morley, CV, et al. 2012. Astrophys. J. Lett. 760:L31

Bulanin, M, Dokuchaev, A, Tonkov, M, Filippov, N. 1984. J. Quant. Spectrosc. Radiat. Transf. $31: 521$ 
Burgasser, AJ, Cruz, KL, Kirkpatrick, JD. 2007. Astrophys. J. 657:494

Burgasser, AJ, Kirkpatrick, JD, Cutri, RM, et al. 2000. Astrophys. J. Lett. 531:L57

Burgasser, AJ, Gillon, M, Faherty, JK, et al. 2014. Astrophys. J. 785:48

Burrows, A, Burgasser, AJ, Kirkpatrick, JD, et al. 2002. Astrophys. J. 573:394

Burrows, A, Hubbard, WB, Lunine, JI, Liebert, J. 2001. Rev. Mod. Phys. 73:719

Burrows, A, Hubbard, WB, Saumon, D, Lunine, JI. 1993. Astrophys. J. 406:158

Burrows, A, Marley, MS, Sharp, CM. 2000. Astrophys. J. 531:438

Burrows, A, Sharp, CM. 1999. Astrophys. J. 512:843

Caffau, E, Ludwig, HG, Steffen, M, Freytag, B, Bonifacio, P. 2011. Sol. Phys. 268:255

Cannon, CJ. 1973. J. Quant. Spectrosc. Radiat. Transf. 13:627

Carbon, DF. 1979. Annu. Rev. Astron. Astrophys. 17:513

Castelli, F, Gratton, RG, Kurucz, RL. 1997. Astron. Astrophys. 318:841

Catling, DC. 2014. Treatises on Geophysics in review

Cattaneo, F, Brummell, NH, Toomre, J, Malagoli, A, Hurlburt, NE. 1991. Astrophys. J. 370:282

Chabrier, G, Baraffe, I. 1997. Astron. Astrophys. 327:1039

Chabrier, G, Baraffe, I, Allard, F, Hauschildt, P. 2000. Astrophys. J. 542:464

Chan, KL, Sofia, S, Wolff, CL. 1982. Astrophys. J. 263:935

Chandrasekhar, S. 1960. Radiative Transfer. Mineola, New York: Dover Publications

Crossfield, IJM, Biller, B, Schlieder, JE, et al. 2014. Nature 505:654

Currie, T, Burrows, A, Itoh, Y, et al. 2011. Astrophys. J. 729:128

Cushing, MC, Marley, MS, Saumon, D, et al. 2008. Astrophys. J. 678:1372

Domoto, GA. 1974. J. Quant. Spectrosc. Radiat. Transf. 14:935

Evans, KF, Stephens, GL. 1991. J. Quant. Spectrosc. Radiat. Transf. 46:413

Fegley, Jr., B, Lodders, K. 1994. Icarus 110:117

-. 1996. Astrophys. J. Lett. 472:L37

Fortney, JJ. 2012. Astrophys. J. Lett. 747:L27

Fortney, JJ, Marley, MS, Lodders, K, Saumon, D, Freedman, R. 2005. Astrophys. J. Lett. 627:L69

Freedman, RS, Lustig-Yaeger, J, Fortney, JJ, et al. 2014. Astrophys. J. Suppl. Ser. submitted

Freedman, RS, Marley, MS, Lodders, K. 2008. Astrophys. J. Suppl. Ser. 174:504

Freytag, B, Allard, F, Ludwig, HG, Homeier, D, Steffen, M. 2010. Astron. Astrophys. 513:A19

Fu, Q, Liou, KN. 1992. J. Atmos. Sci. 49:2139

Fukabori, M, Nakazawa, T, Tanaka, M. 1986. J. Quant. Spectrosc. Radiat. Transf. 36:265

Gabard, T. 2013. J. Mol. Spectrosc. 291:61

Gamache, RR, Hawkins, RL, Rothman, LS. 1990. J. Mol. Spectrosc. 142:205

Gamache, RR, Lamouroux, J. 2013. J. Quant. Spectrosc. Radiat. Transf. 130:158

Gamache, RR, Lynch, R, Brown, LR. 1996. J. Quant. Spectrosc. Radiat. Transf. 56:471

Geballe, TR, Saumon, D, Golimowski, DA, et al. 2009. Astrophys. J. 695:844

Gelino, CR, Marley, MS, Holtzman, JA, Ackerman, AS, Lodders, K. 2002. Astrophys. J. 577:433

Gierasch, P, Goody, R. 1968. Planet. Space Sci. 16:615

Gierasch, PJ, Conrath, BJ. 1985. In Recent Advances in Planetary Meteorology, ed. GE Hunt. Cambridge: Cambridge Univ. Press. 121-146

Gillon, M, Triaud, AHMJ, Jehin, E, et al. 2013. Astron. Astrophys. 555:L5

Goody, R, West, R, Chen, L, Crisp, D. 1989. J. Quant. Spectrosc. Radiat. Transf. 42:539

Goody, R, Yung, YL. 1989. Atmospheric Radiation: Theoretical Basis. Oxford: Oxford Univ. Press Griffith, CA, Yelle, RV, Marley, MS. 1998. Science 282:2063

Guillot, T. 2010. Astron. Astrophys. 520:A27

Guillot, T, Gautier, D, Chabrier, G, Mosser, B. 1994. Icarus 112:337

Hanel, RA, Conrath, BJ, Herath, LW, Kunde, VG, Pirraglia, JA. 1981. J. Geophys. Res. 86:8705

Hansen, BMS. 2008. Astrophys. J. Suppl. Ser. 179:484

Hansen, CJ, Kawaler, SD, Trimble, V. 2004. Stellar Interiors: Physical Principles, Structure, and

Evolution, Astronomy and Astrophysics Library New York: Springer 
Hansen, JE. 1969. Astrophys. J. 155:565

Hauschildt, P. 1992. J. Quant. Spectrosc. Radiat. Transf. 47:433

Hauschildt, PH, Lowenthal, DK, Baron, E. 2001. Astrophys. J. Suppl. Ser. 134:323

Heinze, AN, Metchev, S, Apai, D, et al. 2013. Astrophys. J. 767:173

Helling, C, Casewell, S. 2014. Astron. Astrophys. Rev. in review

Helling, C, Dehn, M, Woitke, P, Hauschildt, PH. 2008a. Astrophys. J. Lett. 675:L105

Helling, C, Oevermann, M, Lüttke, MJH, Klein, R, Sedlmayr, E. 2001. Astron. Astrophys. 376:194

Helling, C, Woitke, P. 2006. Astron. Astrophys. 455:325

Helling, C, Woitke, P, Thi, WF. 2008b. Astron. Astrophys. 485:547

Helling, C, Ackerman, A, Allard, F, et al. 2008c. Mon. Not. R. Astron. Soc. 391:1854

Henyey, L, Vardya, MS, Bodenheimer, P. 1965. Astrophys. J. 142:841

Huang, X, Yung, YL. 2004. J. Atmos. Sci. 61

Hubbard, WB, Burrows, A, Lunine, JI. 2002. Annu. Rev. Astron. Astrophys. 40:103

Hubbard, WB, Smoluchowski, R. 1973. Space Sci. Rev. 14:599

Hubeny, I. 2003. In Astronomical Society of the Pacific Conference Series, Vol. 288, Stellar Atmosphere Modeling, ed. I Hubeny, D Mihalas, K Werner. San Francisco: Astron. Soc. Pacific. 17

Hubeny, I, Burrows, A. 2007. Astrophys. J. 669:1248

Hubeny, I, Lanz, T. 1992. Astron. Astrophys. 262:501

Hubeny, I, Lanz, T. 1995. Astrophys. J. 439:875

Husser, TO, Wende-von Berg, S, Dreizler, S, et al. 2013. Ann. Rev. Astron. ES Astrophys. 553:A6

Kippenhahn, R, Weigert, A, Weiss, A. 2012. Stellar Structure and Evolution, Astronomy and Astrophysics Library New York: Springer

Kirkpatrick, JD. 2005. Annu. Rev. Astron. Astrophys. 43:195

Kondratyev, KY. 1965. Radiative heat exchange in the atmosphere. Oxford: Pergamon Press

Kurucz, RL. 1996. In Astronomical Society of the Pacific Conference Series, Vol. 108, M.A.S.S., Model Atmospheres and Spectrum Synthesis, ed. SJ Adelman, F Kupka, WW Weiss. San Francisco: Astron. Soc. Pacific. 2

Lacis, AA, Hansen, J. 1974. J. Atmos. Sci. 31:118

Lacis, AA, Oinas, V. 1991. J. Geophys. Res. 96:9027

Le Moal, MF, Severin, F. 1986. J. Quant. Spectrosc. Radiat. Transf. 35:145

Leconte, J, Chabrier, G. 2012. Astron. Astrophys. 540:A20

Lee, JM, Fletcher, LN, Irwin, PGJ. 2012. Mon. Not. R. Astron. Soc. 420:170

Lee, JM, Heng, K, Irwin, PGJ. 2013. Astrophys. J. 778:97

Lenzuni, P, Chernoff, DF, Salpeter, EE. 1991. Astrophys. J. Suppl. Ser. 76:759

Line, MR, Fortney, JJ, Marley, MS, Sorahana, S. 2014. ArXiv e-prints arXiv:1403.6412

Line, MR, Zhang, X, Vasisht, G, et al. 2012. Astrophys. J. 749:93

Line, MR, Wolf, AS, Zhang, X, et al. 2013. Astrophys. J. 775:137

Liou, KN. 1974. J. Appl. Meteorol. 31:522

Lodders, K. 1999. Astrophys. J. 519:793

-. 2003. Astrophys. J. 591:1220

Lodders, K. 2004. Science 303:323

Lodders, K. 2010. In Formation and Evolution of Exoplanets, ed. R Barnes. Hoboken, New Jersey: Wiley-VCH. 157

Lodders, K, Fegley, B. 2002. Icarus 155:393

Lodders, K, Fegley, Jr., B. 2006. In Astrophysics Update 2, ed. JW Mason. New York: SpringerPraxis. 1

Ludwig, HG, Allard, F, Hauschildt, PH. 2006. Astron. Astrophys. 459:599

Lunine, JI, Hubbard, WB, Burrows, A, Wang, YP, Garlow, K. 1989. Astrophys. J. 338:314

Lunine, JI, Hubbard, WB, Marley, MS. 1986. Astrophys. J. 310:238

Lupu, R, Zahnle, K, Marley, MS, et al. 2014. Astrophys. J. 784:27 
Macintosh, BA, Graham, JR, Palmer, DW, et al. 2008. Soc. Photo-Opt. Instru. Eng. (SPIE) 7015 Madhusudhan, N, Seager, S. 2009. Astrophys. J. 707:24

Madhusudhan, N, Harrington, J, Stevenson, KB, et al. 2011. Nature 469:64

Manabe, S, Strickler, RF. 1964. J. Atmos. Sci. 21:361

Manabe, S, Wetherald, RT. 1967. J. Atmos. Sci. 24:241

Margolis, JS. 1993. J. Quant. Spectrosc. Radiat. Transf. 50:431

-. 1996. J. Quant. Spectrosc. Radiat. Transf. 55:823

Marley, MS. 2000. In Astronomical Society of the Pacific Conference Series, Vol. 212, From Giant Planets to Cool Stars, ed. CA Griffith MS Marley. San Francisco: Astron. Soc. Pacific. 152

Marley, MS, Ackerman, AS, Cuzzi, JN, Kitzmann, D. 2013. In Comparative Climatology of Terrestrial Planets, ed. SJ Mackwell, AA Simon-Miller, JW Harder, MA Bullock. Tucson: Univ. Arizona Press. 391

Marley, MS, McKay, CP. 1999. Icarus 138:268

Marley, MS, Saumon, D, Cushing, M, et al. 2012. Astrophys. J. 754:135

Marley, MS, Saumon, D, Goldblatt, C. 2010. Astrophys. J. Lett. 723:L117

Marley, MS, Saumon, D, Guillot, T, et al. 1996. Science 272:1919

Marley, MS, Seager, S, Saumon, D, et al. 2002. Astrophys. J. 568:335

McClatchey, RA, Benedict, WS, Clough, SA, Burch, DE, Calfee, RF. 1973. Atmospheric Absorption

Line Parameters Compilation. Tech. Rep. AFCRL-TR-0096. Air Force Cambridge Res. Lab.

McKay, CP, Pollack, JB, Courtin, R. 1989. Icarus 80:23

Meador, W, Weaver, W. 1980. J. Atmos. Sci. 37:630

Meadows, VS, Crisp, D. 1996. J. Geophys. Res. 101:4595

Mihalas, D. 1970. Stellar Atmospheres, Books in Astronomy and Astrophysics London: W. H.

Freeman and Company

Milkey, RW, Shine, RA, Mihalas, D. 1975. Astrophys. J. 202:250

Mlawer, EJ, Payne, VH, Moncet, JL, et al. 2012. R. Soc. London Phil. Trans. Series A 370:2520

Mlawer, EJ, Taubman, SJ, Brown, PD, Iacono, MJ, Clough, SA. 1997. J. Geophys. Res. 102:16663

Morley, CV, Fortney, JJ, Marley, MS, et al. 2012. Astrophys. J. 756:172

Morley, CV, Marley, MS, Fortney, JJ, Lupu, R. 2014a. Astrophys. J. Lett. 789:L14

Morley, CV, Marley, MS, Fortney, JJ, et al. 2014b. Astrophys. J. 787:78

Moses, J. 2000. In Astronomical Society of the Pacific Conference Series, Vol. 212, From Giant

Planets to Cool Stars, ed. CA Griffith MS Marley. San Francisco: Astron. Soc. Pacific. 196

Niemann, HB, Atreya, SK, Carignan, GR, et al. 1998. J. Geophys. Res. 103:22831

Noll, KS, Geballe, TR, Marley, MS. 1997. Astrophys. J. Lett. 489:L87

Olson, GL, Auer, L, Buchler, JR. 1986. J. Quant. Spectrosc. Radiat. Transf. 35:431

Oppenheimer, BR, Kulkarni, SR, Matthews, K, van Kerkwijk, MH. 1998. Astrophys. J. 502:932

Parmentier, V, Guillot, T. 2014. Astron. Astrophys. 562:A133

Pearl, JC, Conrath, BJ, Hanel, RA, Pirraglia, JA, Coustenis, A. 1990. Icarus 84:12

Pecker, JC. 1965. Annu. Rev. Astron. Astrophys. 3:135

Pierrehumbert, RT. 2010. Principles of Planetary Climate. Cambridge: Cambridge Univ. Press

Pollack, JB, Rages, K, Baines, KH, et al. 1986. Icarus 65:442

Pollack, JB, Dalton, JB, Grinspoon, D, et al. 1993. Icarus 103:1

Prandtl, L. 1925. Z. Angew. Math. Mech. 5:136

Prinn, RG, Barshay, SS. 1977. Science 198:1031

Radigan, J, Jayawardhana, R, Lafrenière, D, et al. 2012. Astrophys. J. 750:105

Rice, EL, Barman, T, Mclean, IS, Prato, L, Kirkpatrick, JD. 2010. Astrophys. J. Suppl. Ser. 186:63

Richard, C, Gordon, IE, Rothman, LS, et al. 2012. J. Quant. Spectrosc. Radiat. Transf. 113:1276

Robinson, TD, Catling, DC. 2012. Astrophys. J. 757:104

Robinson, TD, Marley, MS. 2014. Astrophys. J. 785:158

Rodgers, C. 2000. Inverse Methods for Atmospheric Sounding: Theory and Practice, Series on atmospheric, oceanic and planetary physics Singapore: World Scientific 
Rodgers, CD. 1976. Rev. Geophys. Space Phys. 14:609

Rothman, L, Gamache, R, Goldman, A, et al. 1987. Appl. Opt. 26:4058

Rothman, L, Gordon, I, Barber, R, et al. 2010. J. Quant. Spectrosc. Radiat. Transf. 111:2139

Rothman, L, Gordon, I, Babikov, Y, et al. 2013. J. Quant. Spectrosc. Radiat. Transf. 130:4

Rothman, LS, Wattson, R, Gamache, R, Schroeder, JW, McCann, A. 1995. Proc. Soc. Photo Optical Instrumentation Engineers 2471:105

Satoh, M. 2004. Atmospheric circulation dynamics and general circulation models. New York: Springer-Praxis

Saumon, D, Bergeron, P, Lunine, JI, Hubbard, WB, Burrows, A. 1994. Astrophys. J. 424:333

Saumon, D, Geballe, TR, Leggett, SK, et al. 2000. Astrophys. J. 541:374

Saumon, D, Marley, MS. 2008. Astrophys. J. 689:1327

Saumon, D, Marley, MS, Cushing, MC, et al. 2006. Astrophys. J. 647:552

Schreier, F. 2011. J. Quant. Spectrosc. Radiat. Transf. 112:1010

Schwarzschild, K. 1906. Math.-phys. Klasse 195:41

Sharp, CM, Burrows, A. 2007. Astrophys. J. Suppl. Ser. 168:140

Showman, AP, Kaspi, Y. 2013. Astrophys. J. 776:85

Showman, AP, Menou, K, Cho, JYK. 2008. In Astronomical Society of the Pacific Conference Series, Vol. 398, Extreme Solar Systems, ed. D Fischer, FA Rasio, SE Thorsett, A Wolszczan. San Francisco: Astron. Soc. Pacific. 419-441

Smith, MD. 1998. Icarus 132:176

Sorahana, S, Suzuki, TK, Yamamura, I. 2014. Mon. Not. R. Astron. Soc. 440:3675

Sorahana, S, Yamamura, I. 2012. Astrophys. J. 760:151

Spiegel, EA. 1971. Annu. Rev. Astron. Astrophys. 9:323

Stamnes, K, Swanson, RA. 1981. J. Atmos. Sci. 38:387

Stamnes, K, Tsay, SC, Wiscombe, W, Jayaweera, K. 1988. Appl. Optics 27:2502

Stamnes, K, Tsay, SC, Wiscombe, W, Laszlo, I. 2000. NASA Report

Stephens, DC, Leggett, SK, Cushing, MC, et al. 2009. Astrophys. J. 702:154

Stevenson, DJ, Salpeter, EE. 1976. In Jupiter: Studies of the Interior, Atmosphere, Magnetosphere and Satellites, ed. T Gehrels. Tucson: Univ. Arizona Press. 85-112

Tennyson, J, Yurchenko, SN. 2012. Mon. Not. R. Astron. Soc. 425:21

Tinney, CG, Tolley, AJ. 1999. Mon. Not. R. Astron. Soc. 304:119

Toon, OB, McKay, CP, Ackerman, TP, Santhanam, K. 1989. J. Geophys. Res. 94:16287

Tsuji, T. 1971. Pub. Astron. Soc. Jpn. 23:553

-. 1984. Astron. Astrophys. 134:24

Tsuji, T. 1994. In Lecture Notes in Physics, Vol. 428, IAU Colloqium 146: Molecules in the Stellar Environment, ed. UG Jorgensen. New York: Springer. 79-97

—. 2002. Astrophys. J. 575:264

-. 2005. Astrophys. J. 621:1033

Tsuji, T, Ohnaka, K, Aoki, W, Nakajima, T. 1996. Astron. Astrophys. 308:L29

Twomey, S, Jacobowitz, H, Howell, HB. 1966. J. Atmos. Sci. 23:289

van de Hulst, HC. 1963. NASA Report

van Zeggeren, F, Storey, SH. 1970. The Computation of Chemical Equilibria. Cambridge: Cambridge Univ. Press

Vardya, MS. 1970. Annu. Rev. Astron. Astrophys. 8:87

Visscher, C, Lodders, K, Fegley, Jr., B. 2006. Astrophys. J. 648:1181

Vitense, E. 1953. Zeitschrift für Astrophysik 32:135

West, R, Crisp, D, Chen, L. 1990. J. Quant. Spectrosc. Radiat. Transf. 43:191

Winters, BH, Silverman, S, Benedict, WS. 1964. J. Quant. Spectrosc. Radiat. Transf. 4:527

Wiscombe, WJ. 1976. J. Quant. Spectrosc. Radiat. Transf. 16:477

Witte, S, Helling, C, Barman, T, Heidrich, N, Hauschildt, PH. 2011. Astron. Astrophys. 529:A44

Wriedt, T. 2012. In The Mie Theory, ed. W Hergert T Wriedt, Springer Series in Optical Sciences 
New York: Springer. 53-71

Yamamoto, G, Tanaka, M, Asano, S. 1970. J. Atmos. Sci. 27:282

Zahnle, K, Marley, M. 2014. Astrophys. J.

Zahnle, K, Marley, MS, Freedman, RS, Lodders, K, Fortney, JJ. 2009. Astrophys. J. Lett. 701:L20

Zhang, X, Showman, AP. 2014. Astrophys. J. Lett. 788:L6 
Table 1. Selected Elemental Abundances and Species of Interest

\begin{tabular}{|c|c|c|}
\hline Element & $\begin{array}{l}\text { Relative Abundance }^{1} \\
\log [n(\mathrm{El}) / n(\mathrm{H})]+12\end{array}$ & $\begin{array}{l}\text { Important Species } \\
\text { (rainout chemistry) } \\
\end{array}$ \\
\hline $\mathrm{H}$ & 12 & $\mathrm{H}_{2}, \mathrm{H}$ \\
\hline $\mathrm{He}$ & 10.9 & $\mathrm{He}$ \\
\hline $\mathrm{O}$ & 8.7 & $\mathrm{CO}, \mathrm{H}_{2} \mathrm{O}(\mathrm{v}, \mathrm{s}, \mathrm{l})$, silicates \\
\hline $\mathrm{C}$ & 8.4 & $\mathrm{CO}, \mathrm{CH}_{4}, \mathrm{CO}_{2}$ \\
\hline $\mathrm{N}$ & 7.8 & $\mathrm{~N}_{2}, \mathrm{NH}_{3}(\mathrm{v}, \mathrm{s}), \mathrm{NH}_{4} \mathrm{SH}(\mathrm{s})$ \\
\hline $\mathrm{Mg}$ & 7.6 & $\mathrm{MgSiO}_{3}(\mathrm{~s}), \mathrm{Mg}_{2} \mathrm{SiO}_{4}(\mathrm{~s}), \mathrm{MgH}$ \\
\hline $\mathrm{Fe}$ & 7.5 & $\mathrm{FeH}, \mathrm{Fe}(1, \mathrm{~s})$ \\
\hline $\mathrm{S}$ & 7.2 & $\mathrm{H}_{2} \mathrm{~S}, \mathrm{NH}_{4} \mathrm{SH}(\mathrm{s}), \mathrm{MnS}(\mathrm{s}), \mathrm{Na}_{2} \mathrm{~S}(\mathrm{~s}), \mathrm{ZnS}(\mathrm{s})$ \\
\hline $\mathrm{Al}$ & 6.5 & $\mathrm{Al}_{2} \mathrm{O}_{3}(\mathrm{~s})$ \\
\hline $\mathrm{Na}$ & 6.3 & $\mathrm{Na}, \mathrm{Na}_{2} \mathrm{~S}(\mathrm{~s})$ \\
\hline $\mathrm{P}$ & 5.5 & $\mathrm{PH}_{3}, \mathrm{P}_{4} \mathrm{O}_{6}$ \\
\hline $\mathrm{K}$ & 5.1 & $\mathrm{~K}, \mathrm{KCl}(\mathrm{s})$ \\
\hline $\mathrm{Ti}$ & 4.9 & $\mathrm{TiO}, \mathrm{CaTiO}_{3}(\mathrm{~s})$ \\
\hline $\mathrm{V}$ & 4.0 & VO, V-oxides \\
\hline \multicolumn{3}{|c|}{$\begin{array}{l}\text { For condensed phases, }(\mathrm{v})=\text { vapor, }(\mathrm{l})=\text { liquid, }(\mathrm{s})=\text { solid. } \\
{ }^{1} \text { Abundances from Lodders }(2003) . \\
{ }^{2} \text { See Lodders }(2010) \text { for a review. }\end{array}$} \\
\hline
\end{tabular}

Table 2. Parameters for Elementary Cloud Model of Selected Condensates

\begin{tabular}{lccc}
\hline \hline Species & \multicolumn{1}{c}{$f$} & $\varphi=f \frac{m_{c}}{m}$ & $T_{\text {cond }}$ at 3 bar \\
\hline $\mathrm{H}_{2} \mathrm{O}$ & $1.2 \times 10^{-3}$ & 9.7 & 265 \\
$\mathrm{KCl}$ & $2.2 \times 10^{-7}$ & $7.1 \times 10^{-3}$ & 820 \\
$\mathrm{Na}_{2} \mathrm{~S}$ & $1.7 \times 10^{-6}$ & $5.8 \times 10^{-2}$ & 1025 \\
$\mathrm{MgSiO}_{3}$ & $5.9 \times 10^{-5}$ & 2.6 & 1685 \\
$\mathrm{Mg}_{2} \mathrm{SiO}_{4}$ & $3.0 \times 10^{-5}$ & 1.8 & 1760 \\
$\mathrm{Fe}$ & $5.3 \times 10^{-5}$ & 1.3 & 1930 \\
$\mathrm{CaTiO}_{3}$ & $1.4 \times 10^{-7}$ & $8.4 \times 10^{-3}$ & 2010 \\
\hline
\end{tabular}




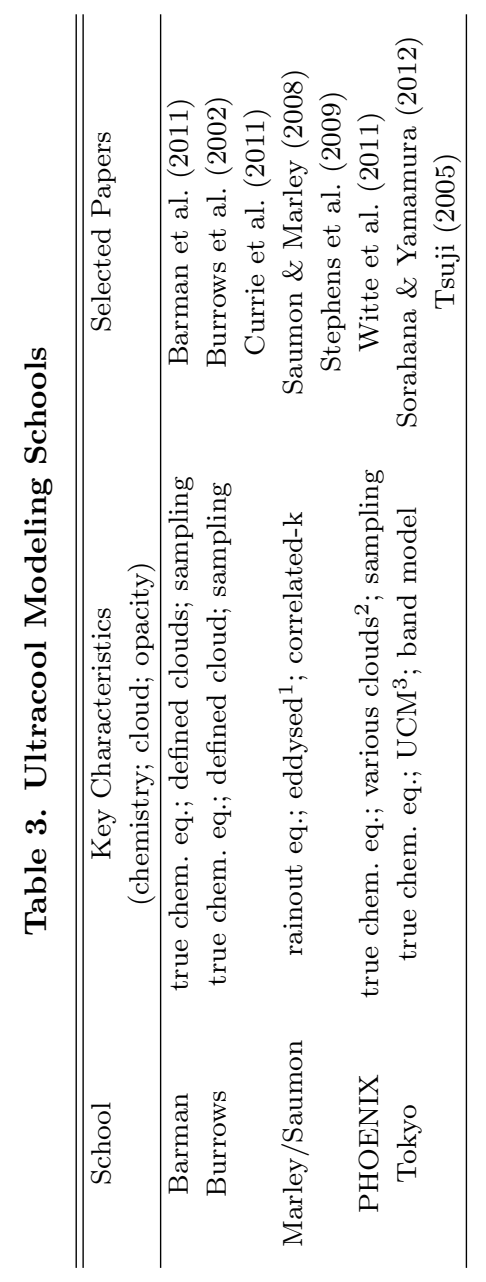

www.annualreviews.org • Brown Dwarf and Giant Planet Atmospheres 
${ }^{1}$ Eddy-sedimentation, a cloud physics model (Ackerman \& Marley 2001).

${ }^{2}$ Various cloud physics models, including DUSTY (Allard et al.|2001) and DRIFT (Witte et al. 2011).

${ }^{3}$ The 'Unified Cloud Model', a defined cloud model (Tsuji 2002). 


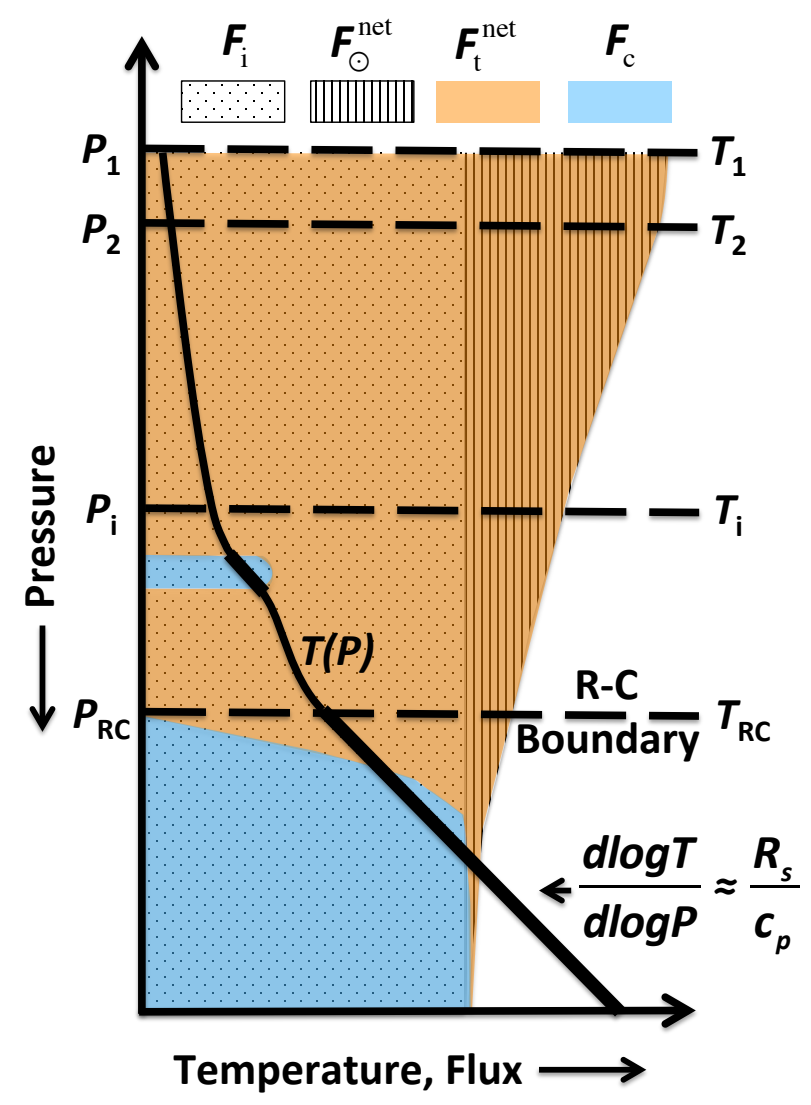

Figure 1

Schematic depiction of a thermal structure model. The vertical axis is pressure, increasing downwards, which is the independent variable, and the horizontal axis shows, relatively, temperature and energy flux. Model levels are shown (horizontal dashed lines), and the solid line is the thermal structure (i.e., temperature) profile, where bolded lengths indicate a convective region. Level pressures and temperatures are indicated with associated sub-scripted symbols, and 'RC' indicates the radiative-convective boundary. In equilibrium, net thermal flux ( $F_{\mathrm{t}}^{\text {net }}$, orange) and the convective flux $\left(F_{\mathrm{c}}\right.$, blue) must sum to the internal heat flux $\left(F_{\mathrm{i}}\right.$, dotted) and, for an irradiated object, the net absorbed stellar flux $\left(F_{\odot}^{\text {net }}\right.$, striped). Note that the internal heat flux is constant throughout the atmosphere, whereas the schematic profile of net absorbed stellar flux decreases with increasing pressure, and eventually reaches zero in the deep atmosphere. At depth, convection carries the vast majority of the summed internal and stellar fluxes, but is a smaller component in detached convective regions (upper blue region). 


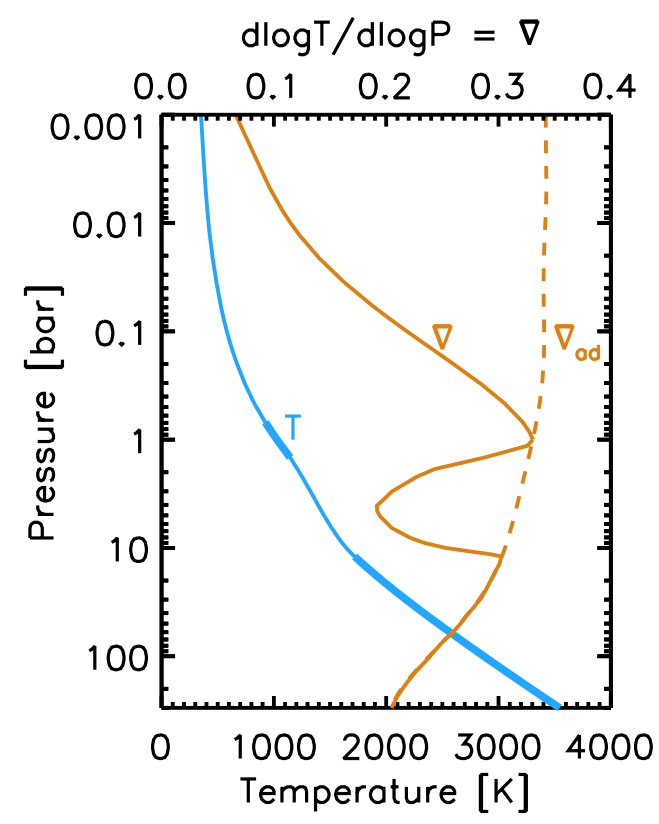

Figure 2

(Two pages.) Illustration of some of the influences on a radiative-convective model, as well as model output. The first panel shows a computed thermal profile (blue), for a cloud-free mid-T dwarf, showing temperature as a function of pressure in the atmosphere. Orange curves compare the computed atmospheric temperature gradient $(\nabla$, solid) with the local adiabatic gradient $\left(\nabla_{\mathrm{ad}}\right.$, dashed). The model has two convection zones (thickened blue), and in both regions $\nabla=\nabla_{\text {ad }}$. The second panel helps illustrate why these two convective zones form - each sub-plot shows, for the indicated pressure level, spectra of the local Planck function (blue), the scaled net thermal flux (orange), and the column absorptivity (dark gray) (i.e, $1-e^{-\tau}$, where $\tau$ is the optical depth between the top-of-atmosphere and the indicated pressure). The absorptivity for $\tau=1$ is shown as a horizontal line in light gray. In the deepest level $(P=100$ bar $)$, convection is carrying the internal heat flux, and the net thermal flux is small. However, near $P=10$ bar, windows in the opacity spectrum align with the local Planck function, thus allowing thermal radiation to carry the internal flux, and the atmosphere forms a deep radiative layer. Further up in the atmosphere, at $P=1$ bar, the local Planck function moves into a region of strong water vapor and methane opacity, thereby re-invigorating convection, which then carries some part of the internal heat flux. Finally, by $P=0.5$ bar, the atmosphere is in strict radiative equilibrium, which remains towards all smaller pressures (or larger altitudes). 


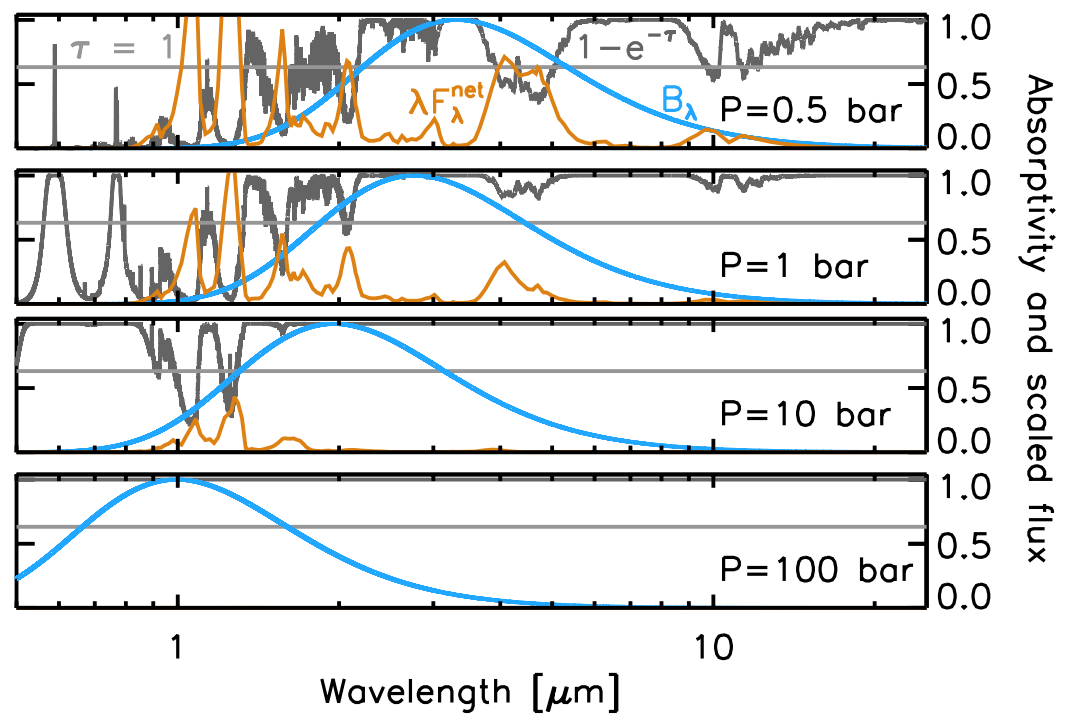



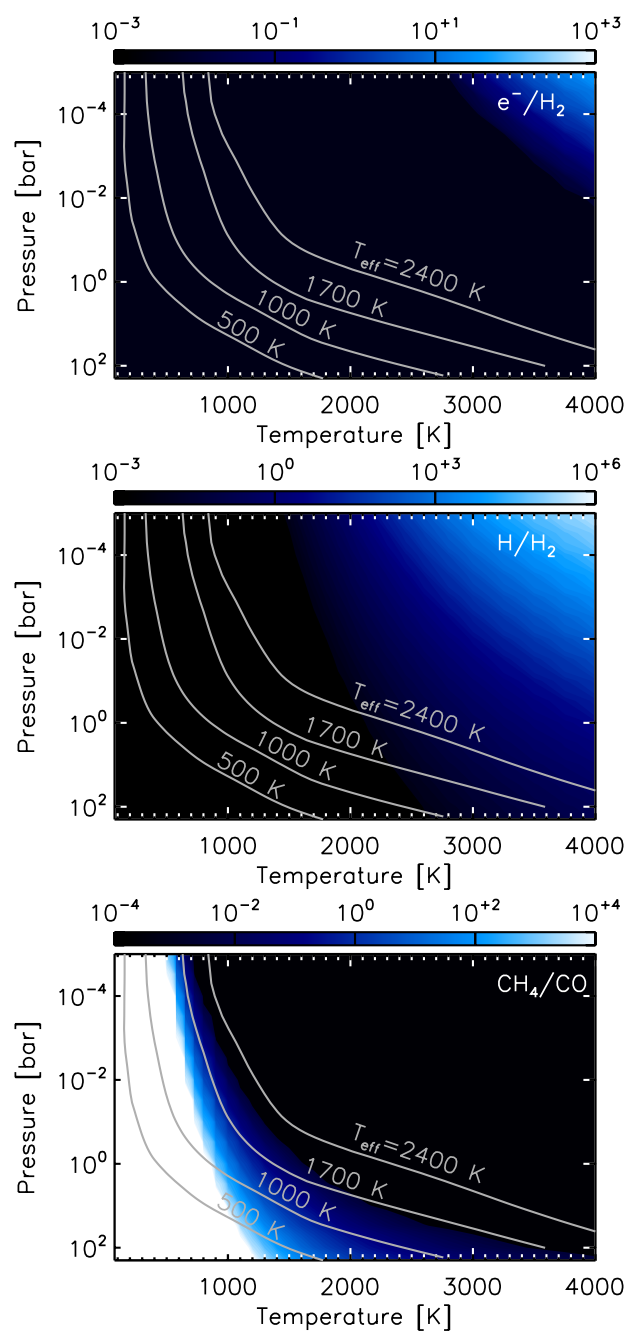

Figure 3

(Two pages.) Abundances or ratios of abundances for various species of interest, as a function of pressure and temperature, from Lodders \& Fegley (2002) and Visscher et al. (2006). Species are listed in the upper-right of each sub-plot, and the abundance, or abundance ratio, is according to the color bar at the top of each sub-plot. Thermal profiles from cloud-free models of various effective temperatures, and with $g=10^{3} \mathrm{~m} \mathrm{~s}^{-2}$, are over-plot in gray. Photospheres for these models typically extend to depths of 3-30 bar. Note the transition from $\mathrm{CO}$ to $\mathrm{CH}_{4}$ at cooler temperatures, and a similar transition for $\mathrm{N}_{2}$ to $\mathrm{NH}_{3}$. Both $\mathrm{TiO}$ and $\mathrm{FeH}$ are strongly depleted by rainout at lower temperatures. 

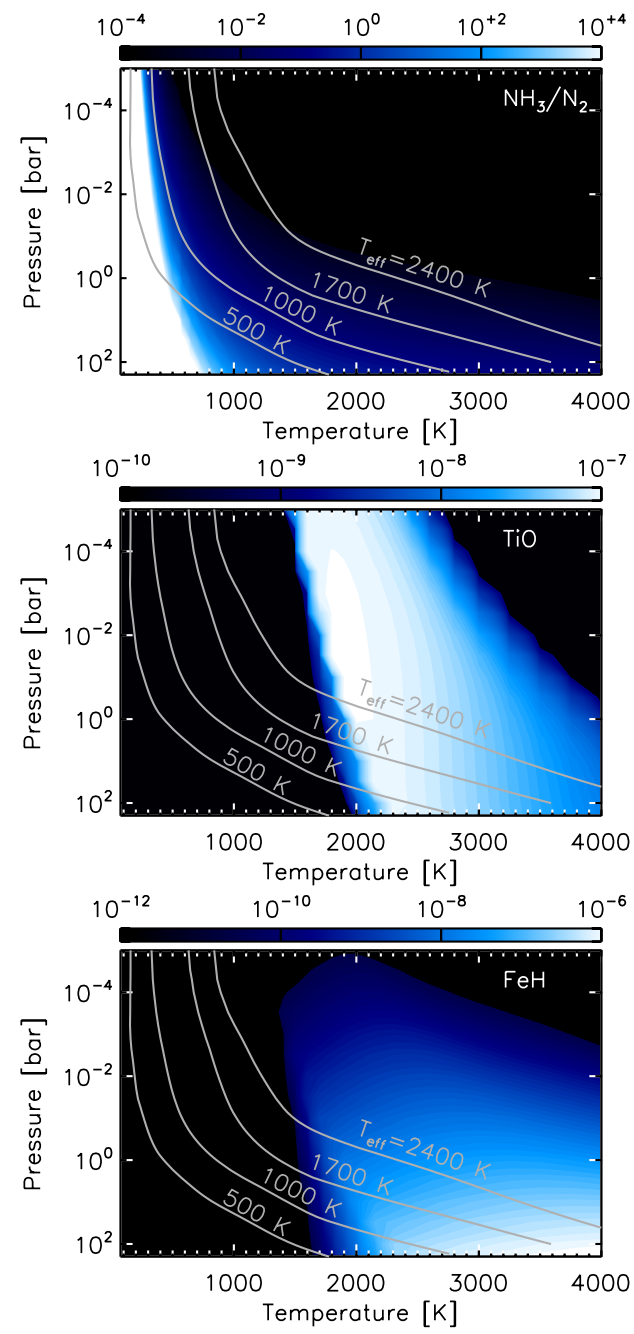


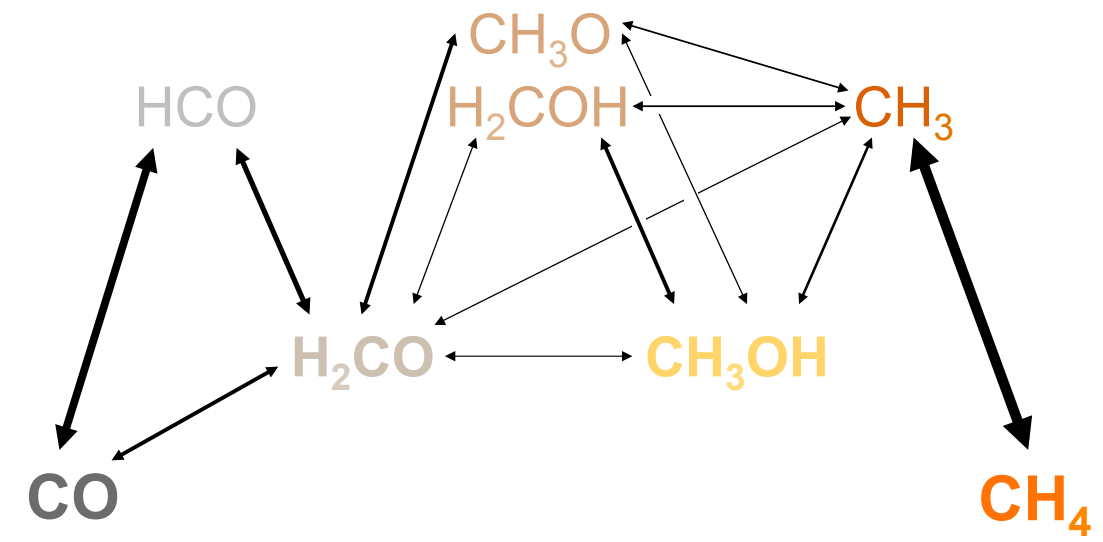

Figure 4

Major chemical pathways linking $\mathrm{CO}$ and $\mathrm{CH}_{4}$ in an $\mathrm{H}_{2}$-rich atmosphere (from Zahnle \& Marley 2014). Reactions from left to right are with $\mathrm{H}_{2}$ or $\mathrm{H}$. Key intermediate molecules are formaldehyde $\left(\mathrm{H}_{2} \mathrm{CO}\right)$ and methanol $\left(\mathrm{CH}_{3} \mathrm{OH}\right)$ while other intermediates $\left(\mathrm{HCO}, \mathrm{H}_{2} \mathrm{COH}, \mathrm{CH}_{3} \mathrm{O}\right.$, $\mathrm{CH}_{3}$ ) are short-lived free radicals. The vertical position of individual species gives a rough indication of the energetics. Energy barriers correspond to breaking $\mathrm{C}-\mathrm{O}$ bonds-from triple to double, from double to single, and from single to freedom. Relative magnitudes of reaction rates are indicated by arrow thickness. Conceptually, higher temperatures and lower pressures tilts the plot as a whole to the left, with carbon pooling in CO. Lower temperatures and higher pressures tilts the plot as a whole to the right, and carbon pools in $\mathrm{CH}_{4}$. 


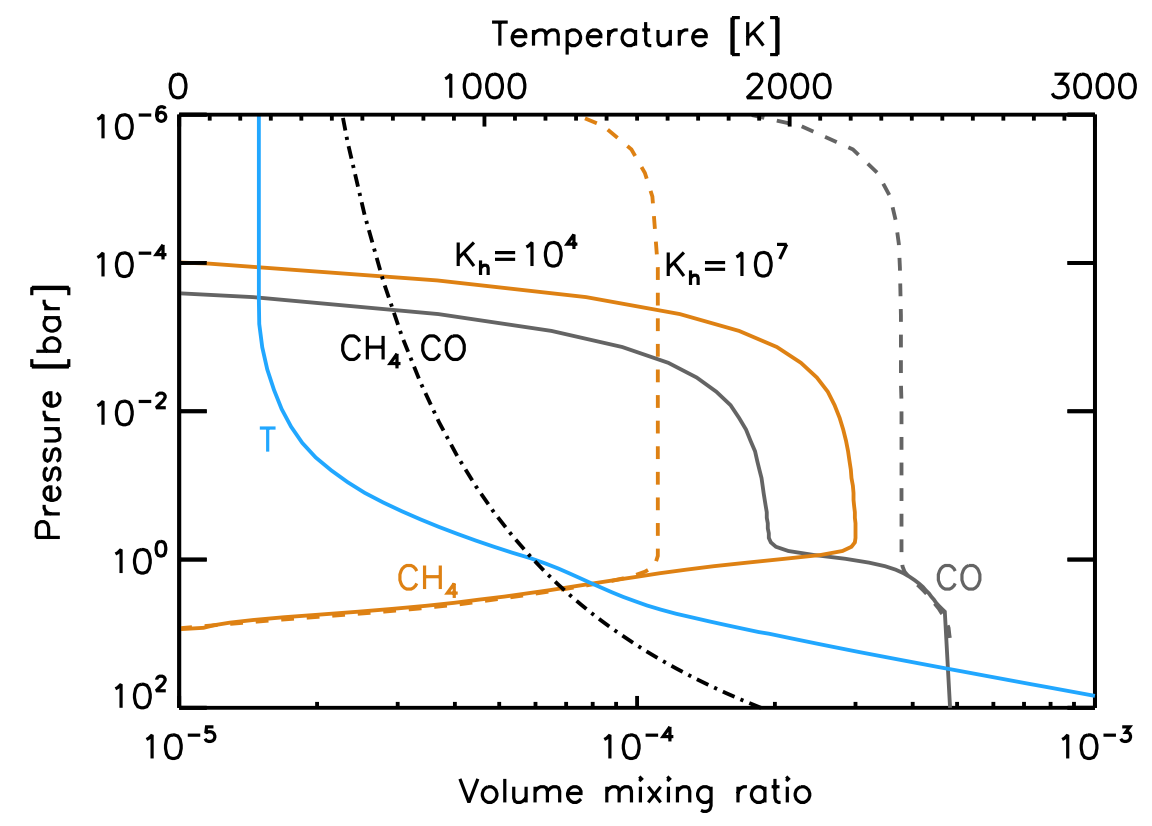

Figure 5

An example of $\mathrm{CH}_{4}$ and $\mathrm{CO}$ disequilibrium. This $\sim 2 \mathrm{M}_{\mathrm{J}}$ giant planet model is cloud-free, has $T=600 \mathrm{~K}, g=10 \mathrm{~m} / \mathrm{s}^{2}$, and no insolation. The $P-T$ profile of the atmosphere (blue) is compared to the $P-T$ curve (black dot-dash) where the mixing ratios of methane and carbon monoxide are equal under equilibrium conditions. Methane is thermodynamically favored when $T$ is to the left of this curve. Disequilibrium $\mathrm{CO}$ (gray) and $\mathrm{CH}_{4}$ (orange) mixing ratios, computed by Zahnle \& Marley (2014), with $K_{h}=10^{4} \mathrm{~cm}^{2} / \mathrm{s}$ (solid) and $K_{h}=10^{7} \mathrm{~cm}^{2} / \mathrm{s}$ (dashed) are shown. Below the quench points, $\mathrm{CO}$ and $\mathrm{CH}_{4}$ are in equilibrium. Above the quench points, the $\mathrm{CO}$ and $\mathrm{CH}_{4}$ mixing ratios are are constant with altitude until molecular diffusion leads to the separation of $\mathrm{CO}$ and $\mathrm{CH}_{4}$ from the background $\mathrm{H}_{2}$ gas at lower pressures. 


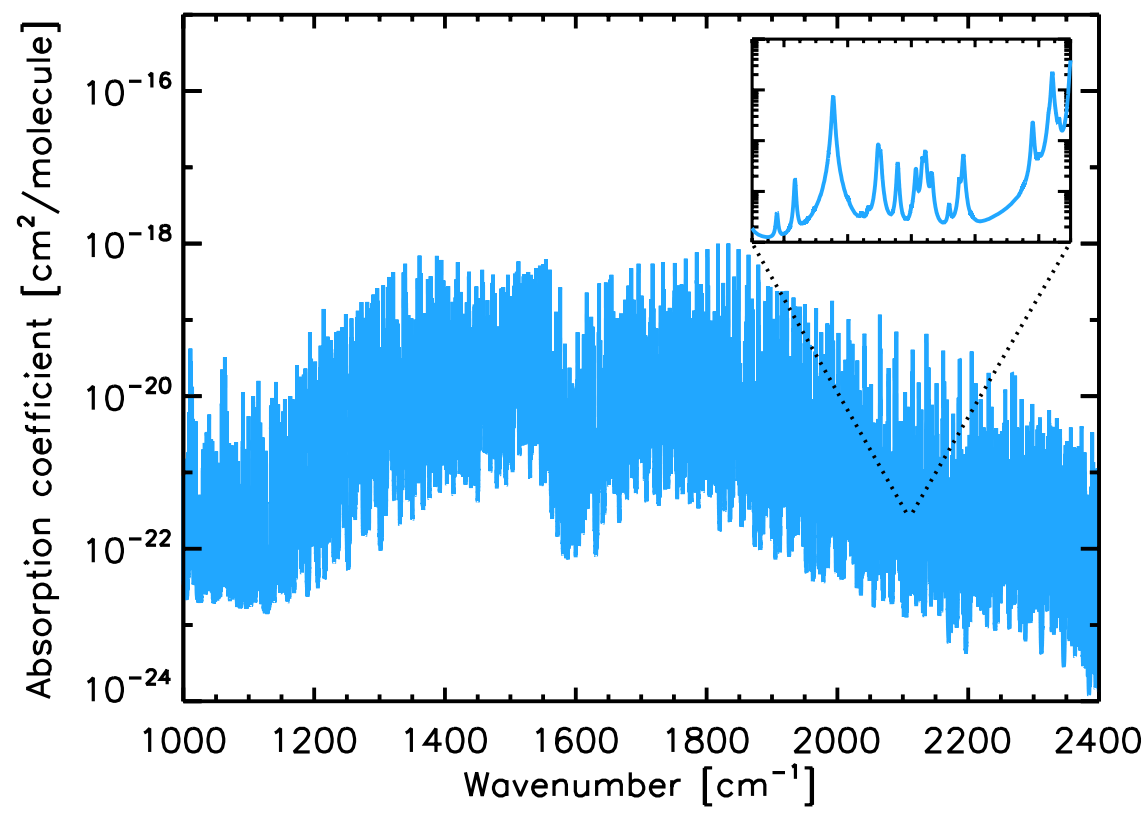

Figure 6

Demonstration of molecular line absorption opacity. Absorption coefficients for the $1600 \mathrm{~cm}^{-1}$ $(6.3 \mu \mathrm{m})$ water vapor absorption band are shown for a temperature of $1,000 \mathrm{~K}$ and a pressure of 1 bar. Inset shows a smaller range, centered at $2100 \mathrm{~cm}^{-1}$ and spanning $10 \mathrm{~cm}^{-1}$, where individual absorption lines can clearly be distinguished. 


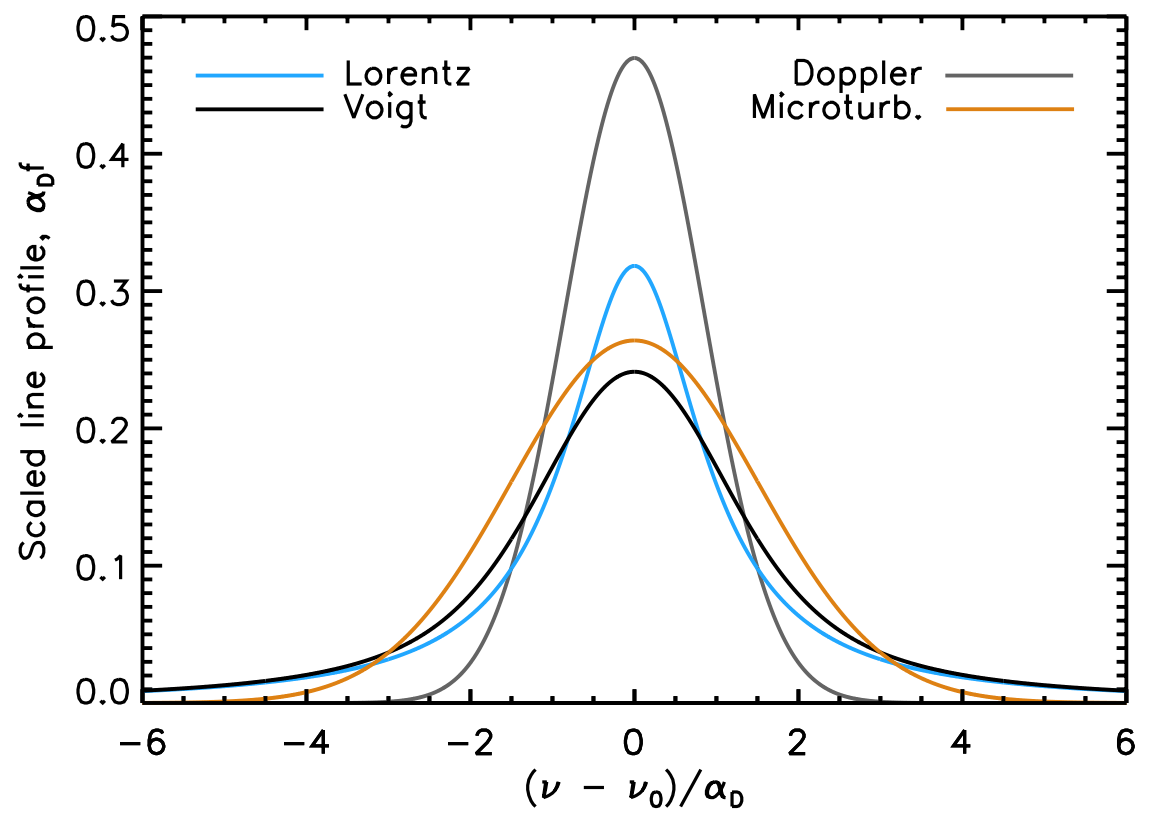

Figure 7

Comparison of different lineshapes. The horizontal axis is in units of the Doppler half-width $\left(\alpha_{D}\right)$, so that a half-width of one occurs at unity along the x-axis. The lineshape, $f$, is scaled by the Doppler half-width, and the Lorentz and Voigt lineshapes use $\alpha_{L}=\alpha_{D}$, so that the area under the curves are all equal (see Huang \& Yung 2004). Also shown is a Doppler lineshape where microturbulent broadening is included, where the turbulent velocity is taken to be $1 \mathrm{~km} \mathrm{~s}^{-1}$. 


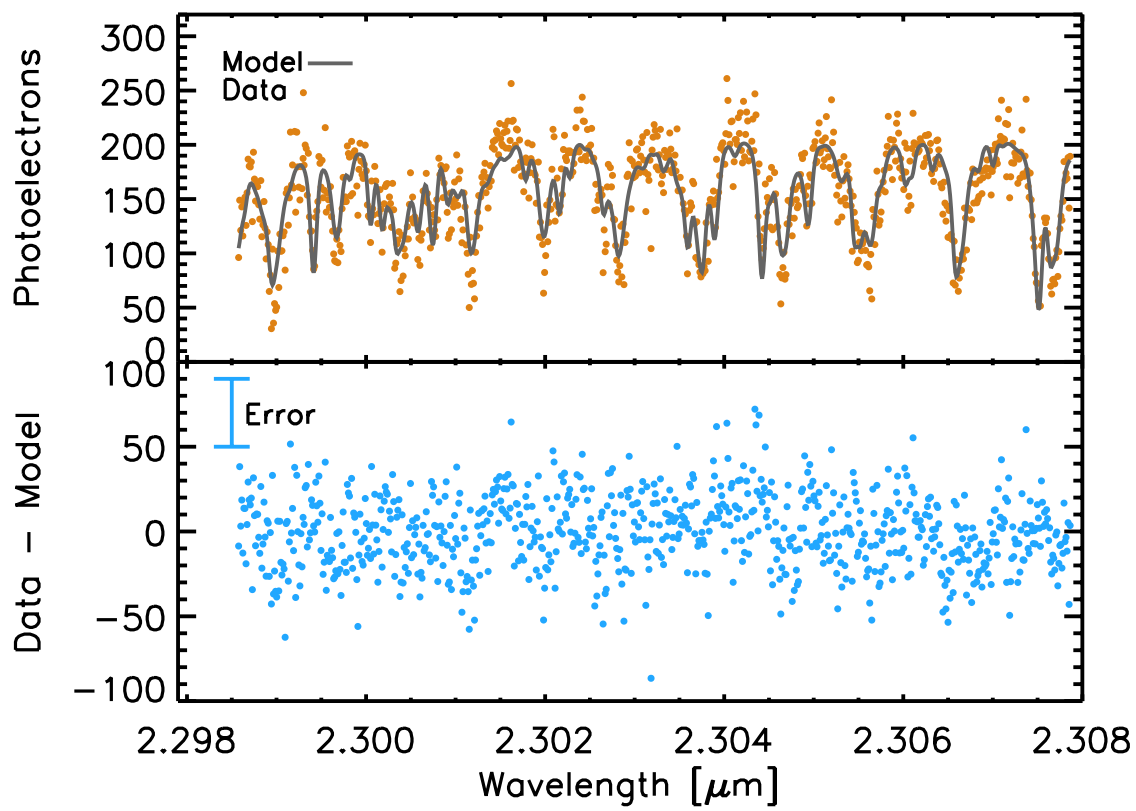

Figure 8

Comparison of a model spectrum to observations of the L2 dwarf 2MASS J01090150-5100494 (from Blake et al. 2007), obtained at a spectral resolution of about $\lambda / \Delta \lambda=50,000$. The top panel shows the observed data as small points (orange) with a best-fit model overplotted as a line (gray, with $T_{\text {eff }}=2200 \mathrm{~K}$ and $g=10^{3} \mathrm{~m} \mathrm{~s}^{-2}$ ). The bottom panel shows the residuals of this fit (blue), and the error bar in the upper left corner approximates the expected noise (photon and read noise) of the spectrum. The agreement indicates that, in this case, the model line shapes (primarily due to $\mathrm{H}_{2} \mathrm{O}$ and $\mathrm{CO}$ lines) do a good, although not perfect, job of reproducing the observations even at this high resolution. 


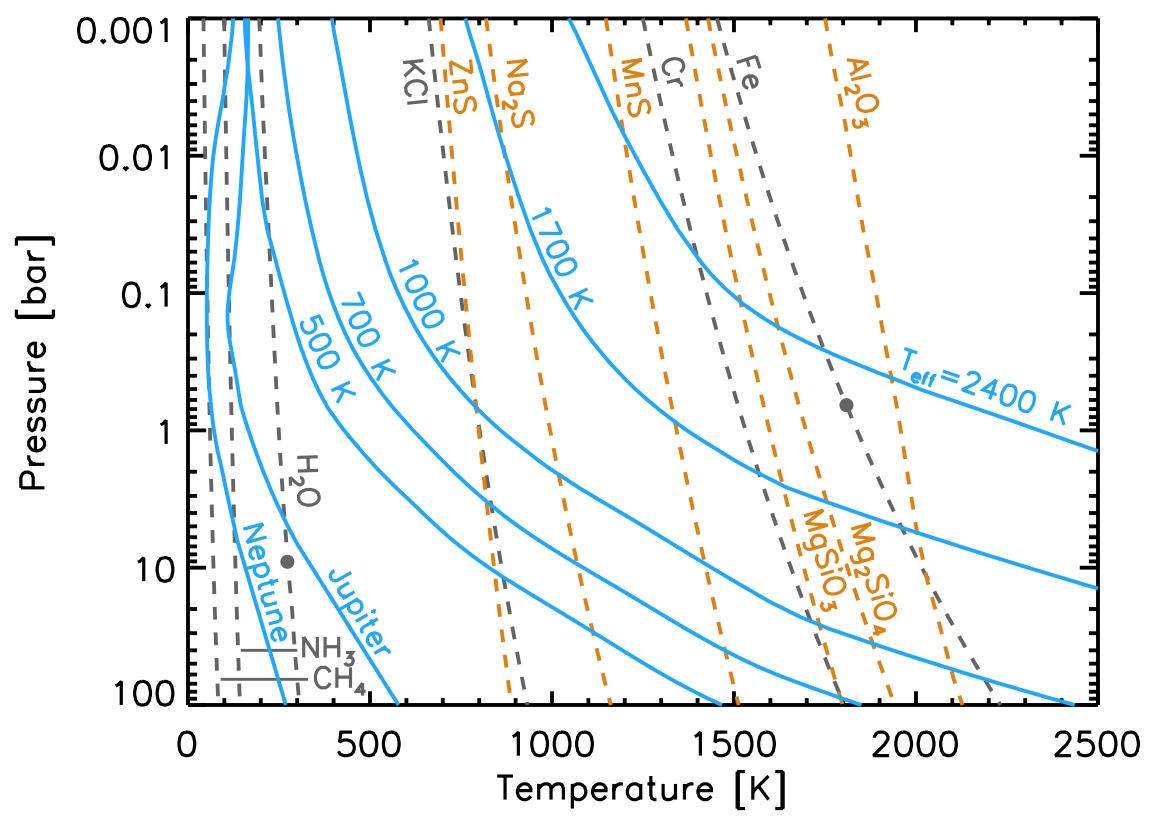

Figure 9

Condensation curves for a variety of species (dashed), assuming solar abundances from Lodders (2003). Gray curves are for direct condensation, while orange curves are for condensates that form due to chemical reactions. Filled circles indicate a liquid-solid transition. Several cloud-free model thermal profiles are provided for comparison, as well as empirically-derived profiles for Jupiter and Neptune. 

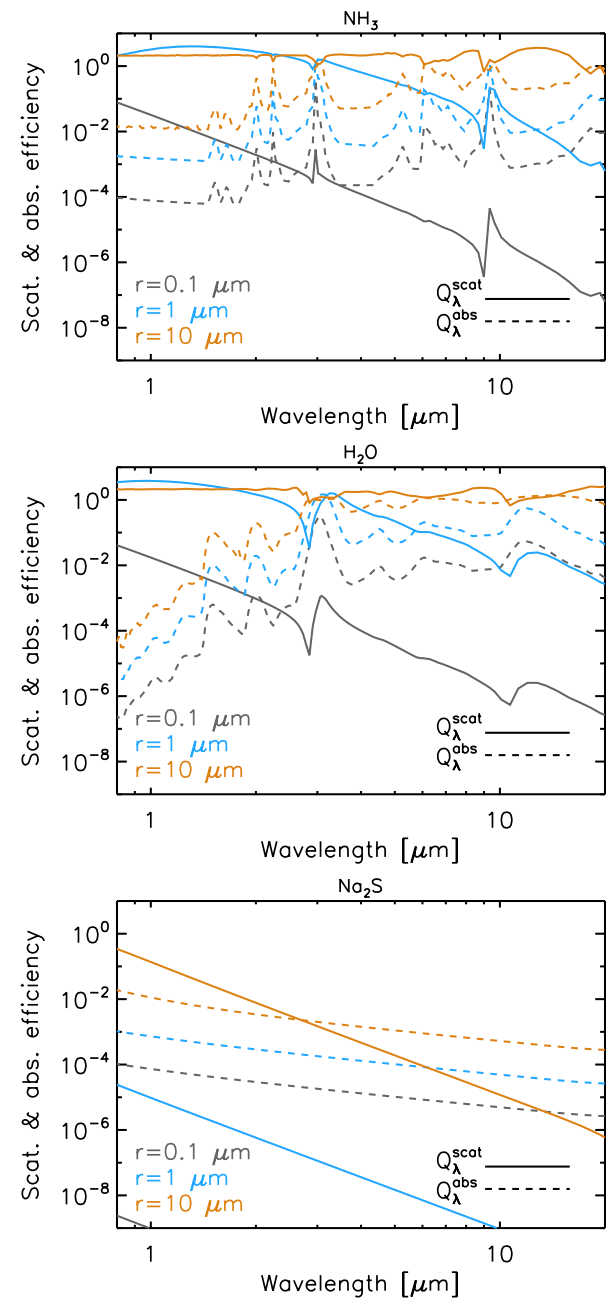

Figure 10

(Two pages.) Absorption and scattering efficiencies for six different condensible species computed using Mie theory. In each panel, the Mie scattering and absorption efficiencies, $Q_{\lambda}^{\text {scat }}$ (solid) and $Q_{\lambda}^{\text {abs }}$ (dashed), are shown for three particle sizes - $0.1 \mu \mathrm{m}$ (black), $1 \mu \mathrm{m}$ (blue), and $10 \mu \mathrm{m}$ (orange). Larger particles are more efficient at both absorbing and scattering for most wavelengths. Figure generalized from Morley et al. (2014b). 

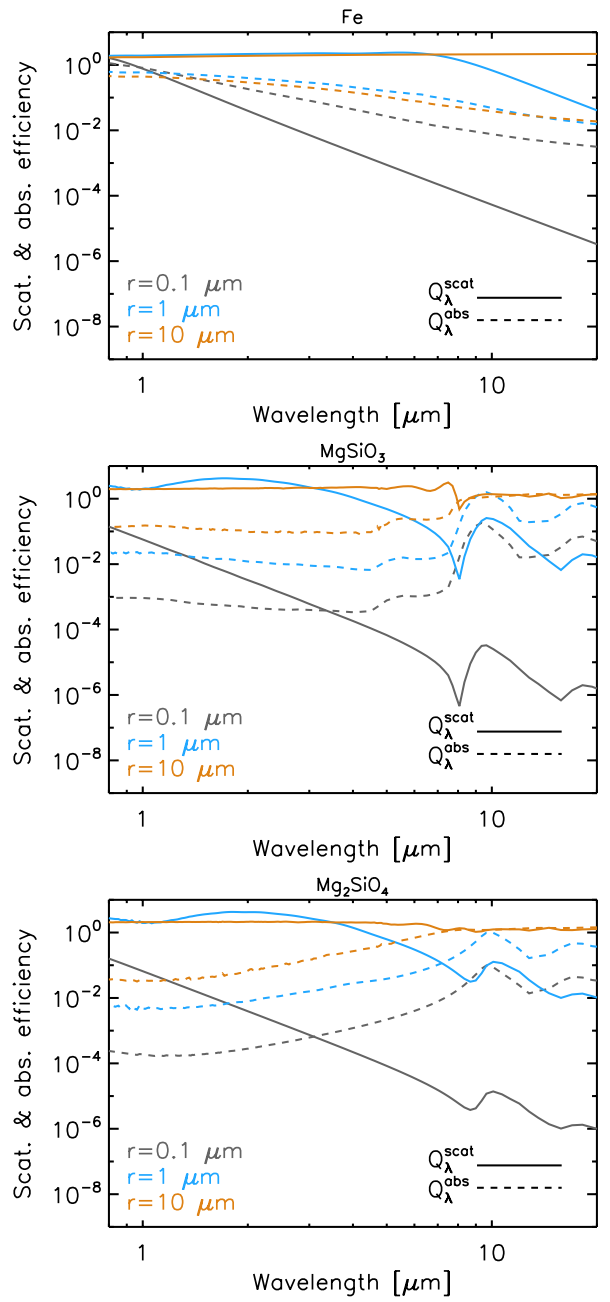

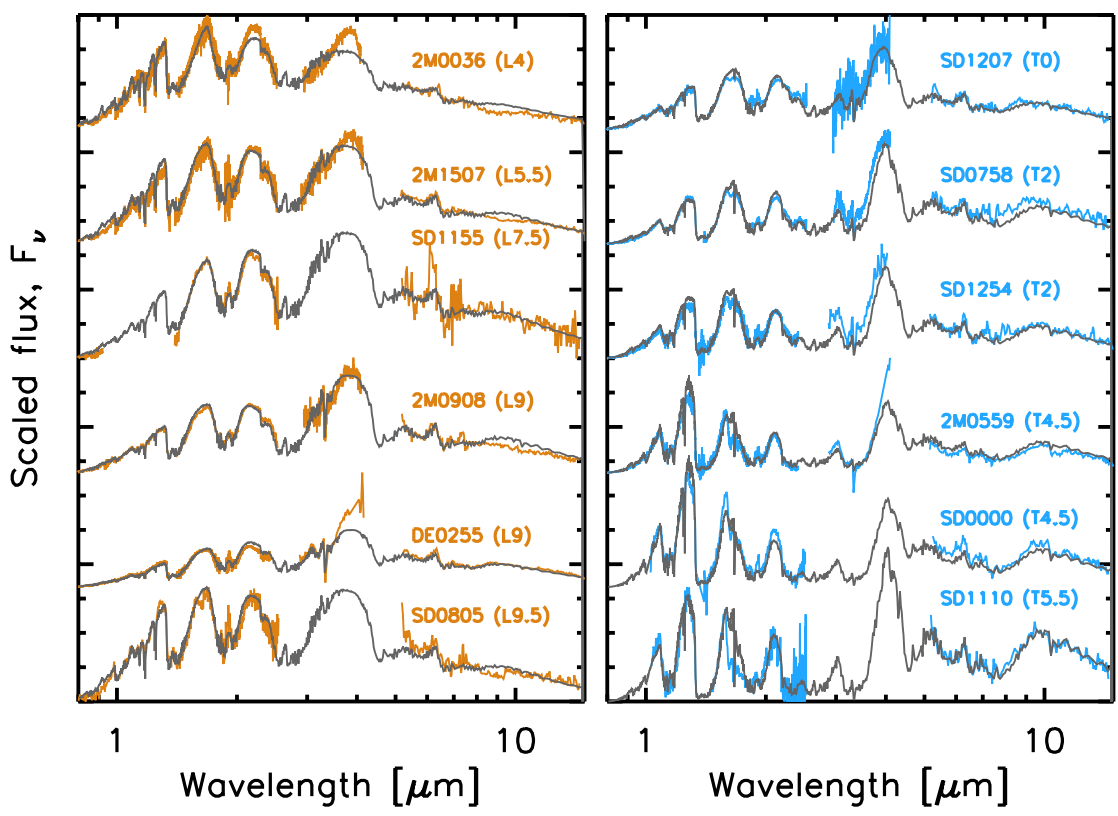

Figure 11

Spectral comparison between observations and best-fit models (gray), after Stephens et al. (2009). The left sub-plot shows L dwarfs (orange), and the right shows T dwarfs (blue). Spectral types are indicated in parentheses. Details regarding data and model properties can be found in Stephens et al. (2009). 


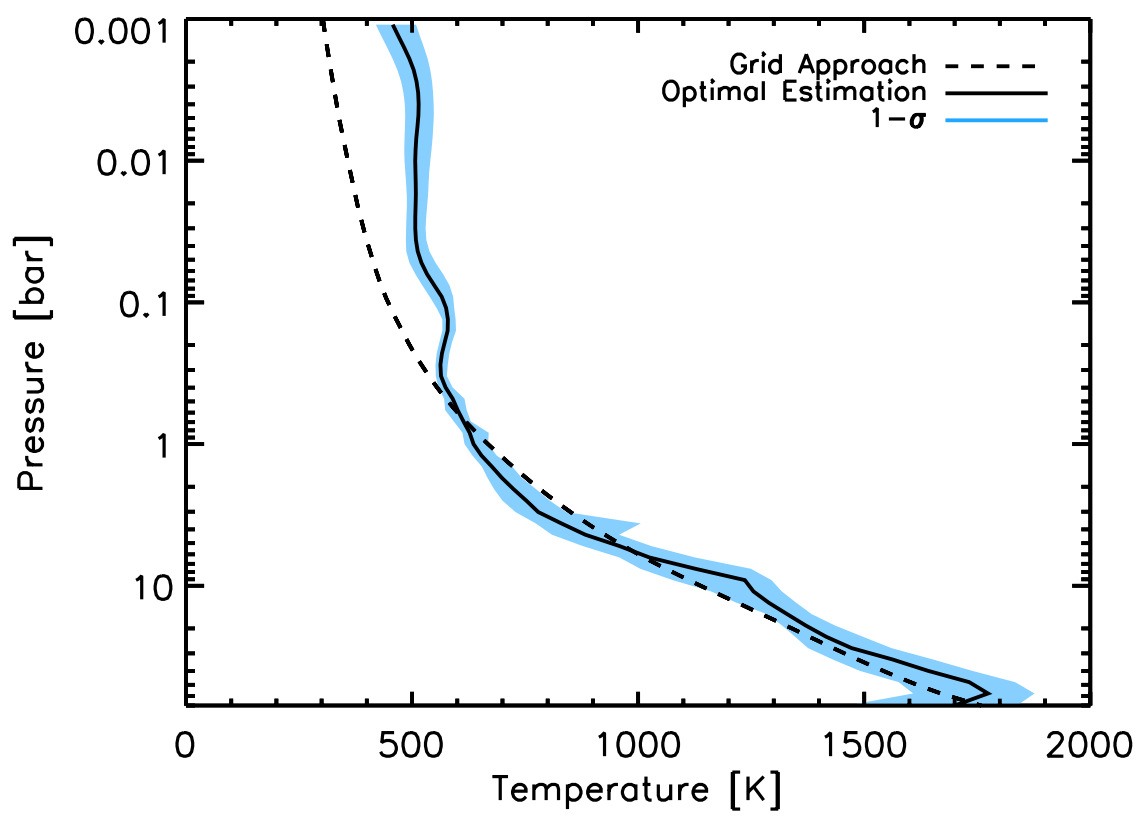

Figure 12

Comparison of two approaches for determining the thermal structure of a brown dwarf, as applied to GL 570D (Burgasser et al. 2000). A grid comparison approach from Saumon et al. (2006) is shown (dashed), and a retrieved $P-T$ profile (solid) with $1-\sigma$ error bars (blue shaded), determined using optimal estimation techniques, are also shown. Figure adapted from Line et al. (2014). 